Communication

\title{
Study on the Fluorescent Activity of $N^{2}$-Indolyl-1,2,3-triazole
}

\author{
You-Can Zhang, Rui Jin, Luo-Yuan Li, Zili Chen * and Li-Min Fu * \\ Department of Chemistry, Renmin University of China, Beijing 100872, China; 2015101964@ruc.edu.cn (Y.-C.Z.); \\ jinrui87@ruc.edu.cn (R.J.); luoyuanli1209@126.com (L.-Y.L.) \\ * Correspondence: zilichen@ruc.edu.cn (Z.C.); lmfu@ruc.edu.cn (L.-M.F.); \\ Tel./Fax: +86-010-6251-2822 (Z.C. \& L.-M.F.)
}

Received: 24 July 2017; Accepted: 15 August 2017; Published: 5 September 2017

\begin{abstract}
A new type of blue emitter, $N^{2}$-Indolyl-1,2,3-triazoles (NITs), with the $\lambda_{\max }$ ranging from $420-480 \mathrm{~nm}$ and the Stokes shift from 89-143 nm, were synthesized through the coupling reaction of indoles with triazole derivatives. The influence of different substitution patterns on the optical properties (efficiency, excitation, and emission wavelengths) of the NITs was investigated. In addition, one palladium complex were synthesized by using NITs as the ligands, which, however, exhibited no fluorescent activity, but did show the enhanced co-planarity. Lastly, two bio-active molecule derivatives were explored for the potential use of these novel dyes in related chemical and biological applications.
\end{abstract}

Keywords: $N^{2}$-indolyl-1,2,3-triazoles (NITs); fluorescent activity; blue-emissive fluorophore; $N^{2}$-aryl-1,2,3-triazoles (NATs)

\section{Introduction}

Photoactive molecules have been the object of many studies in chemistry, biology, and material research [1-5]. A great number of fluorescent compounds emitting different colors (such as red, green, etc.) has been developed over the past years for their utilization in the biological target imaging [6-11], effective photosensors [12-17], and novel photoactive materials [18-23]. However, the corresponding blue-emissive fluorophores, which exhibit blue fluorescence in high quantum efficiency coupled with high stability, are more difficult to obtain [24-28], due to the large energy gaps that exist between the blue emitter's highest occupied molecular orbitals (HOMOs) and its lowest unoccupied molecular orbitals (LUMOs) [29,30].

This optical activity and photostability dilemma is a great challenge for the development of efficient, stable blue-light-emitting small molecular fluorophores. For example, biphenyl-type molecules and similar structures usually have lower photoactivity, because of the poor conjugation and the ortho-substituted group's steric repulsions [31-33]. On the other hand, extended aromatic systems, in which at least one double bond exists between the aromatic groups, have high quantum efficient blue emission. However, the photolabile double bonds limit their potential applications [34-38].

In 2011, Shi and his coworkers reported $N^{2}$-Aryl-1,2,3-triazoles (NATs, Scheme 1 ) as a new type of blue-light emitting fluorophore, featured by its tunable optical emission ( $\lambda_{\max }$ range from $350-400 \mathrm{~nm}$ and Stokes shift from 38-93 nm), with a moderate to good quantum efficiency and a relatively higher stability $[39,40]$. It was proposed that the blue fluorescence of NAT resulted from a planar intramolecular charge transfer (PICT) mechanism. 
<smiles>[R]c1ccc(-n2ncc([R])n2)cc1</smiles>

2011, Shi group (NAT)<smiles>[R][R]1ccc2c(c1)cc(-n1ncc([R2])n1)n2[R]</smiles>

2014, Our group (NIT)

Scheme 1. The structure of N-2-Aryl-1,2,3-triazoles (NATs) \& N-2-Indolyl-1,2,3-triazoles (NITs).

Recently, we developed a similar molecular structure, $N^{2}$-Indolyl-1,2,3-triazole (NITs) through the $N^{2}$-selective coupling of 1,2,3-triazoles with indoles via iodo-mediation, which are also good blue emitters, with the $\lambda_{\max }$ ranging from $420-480 \mathrm{~nm}$ and the Stokes shift from 89-143 nm [41,42]. Starting from this structure, we synthesized a new single-fluorophore-based fluorescent probe for the dual-channel detection of $\mathrm{Ag}^{+}$and $\mathrm{Hg}^{2+}$ ions [43]. As an extension of the previous research, a series of NITs with different substitution patterns were prepared using the method shown in Scheme 2, and the optical properties of these new blue emitters were investigated in this paper. In addition, one palladium complexes were synthesized by using NITs as ligands, which, however, exhibited no fluorescent activity, but did present enhanced co-planarity.

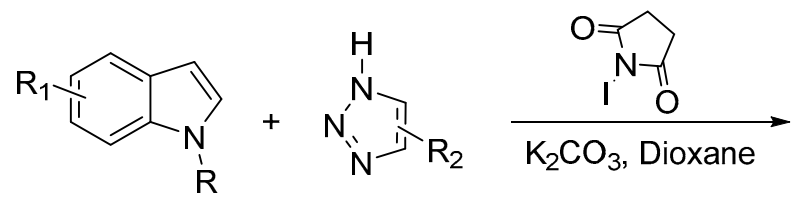<smiles>[R]c1cnn(-c2cc3c(n2[R])C=C[R]C=C3)n1</smiles>

Scheme 2. Method for the preparation of various substituted $N^{2}$-Indolyl-1,2,3-triazole (NITs).

\section{Results and Discussions}

The structures of the NITs allowed modification on three different positions: the indole nitrogen atom ( $R$ group), the triazole moiety ( $R_{1}$ group), and the indole ring $\left(R_{2}\right.$ group) (Scheme 2$)$. To evaluate these new fluorophores, we synthesized compounds $\mathbf{3 a}-\mathbf{y}$ and explored the influence of different substitution patterns on the optical properties (efficiency, excitation, and emission wavelengths) of the NITs.

Influence of the indole $N$-position: At first, the effect of $N$-substituted R group was investigated. It was proposed that the fluorescence activity of NATs the was greatly affected by the co-planarity between the phenyl ring and the triazole group (Scheme 1) [39,40]. We considered that R group in NITs, which was located nearby the rotating $C-N$ bond between the indole ring and the triazole group, would affect the co-planarity of NITs, and thus influence their optical properties.

The absorption and emission behavior of compounds 3a-e are summarized in Table 1 , Figures 1 and 2. A NAT compound, 2,4-diphenyl-2H-1,2,3-triazole (compound 4), was tested as the comparison $[39,40]$, because of its structural similarity with NIT molecules and its synthetic convenience. As shown in Table 1, compound 4 absorbs UV light at $292 \mathrm{~nm}$ and emits blue fluorescence at around $345 \mathrm{~nm}$. It was found that the UV absorbance of NITs 3a-e ranged from $298 \mathrm{~nm}$ to $325 \mathrm{~nm}$. The peaks of their fluorescence emission had an obvious red-shift compared with 4 , which might result from indole group's stronger electro-donor ability compared to the N-phenyl group of NATs, as well as the larger conjugated system. In addition, all NIT compounds' Stokes shifts are larger than that of NAT molecule 4. 
Table 1. Fluorescence emission behavior of compounds with different Indole $N$-substitutions (a).
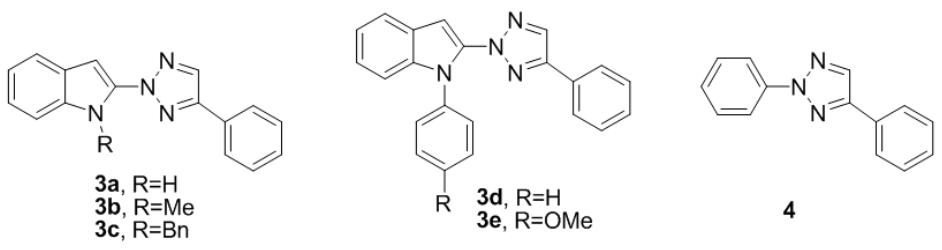

\begin{tabular}{cccccc}
\hline & Absorption $(\mathbf{n m})$ & Emission $\left(\boldsymbol{\lambda}_{\text {max }}\right)(\mathbf{n m})$ & $\boldsymbol{\Phi}^{(\mathbf{b})}$ & Fluorescence Intensity $\left(\times \mathbf{1 0}^{\mathbf{3}}\right)$ & Stokes Shift \\
\hline $\mathbf{4}$ & $292(0.238)$ & 345 & 0.39 & 46.3 & 53 \\
$\mathbf{3 a}$ & $325(0.246)$ & 429 & 0.65 & 28.20 & 104 \\
$\mathbf{3 b}$ & $310(0.238)$ & 437 & 0.90 & 128.77 & 127 \\
$\mathbf{3 c}$ & $315(0.219)$ & 432 & 0.83 & 105.02 & 117 \\
$\mathbf{3 d}$ & $298(0.312)$ & 422 & 0.97 & 136.94 & 124 \\
$\mathbf{3 e}$ & $298(0.258)$ & 423 & 0.99 & 109.48 & 125 \\
\hline
\end{tabular}

(a) Sample information: $1.0 \times 10^{-5} \mathrm{~mol} \mathrm{~L}^{-1}$ in dichloromethane (DCM), excitation at $320 \mathrm{~nm}$ with $2.5 \mathrm{~nm}$ slit.

(b) Quantum yields $(\Phi)$ were determined based on $1.0 \times 10^{-5} \mathrm{~mol} \mathrm{~L}^{-1}$ 9,10-Diphenylanthracene in cyclohexane $(\Phi=0.95)$.

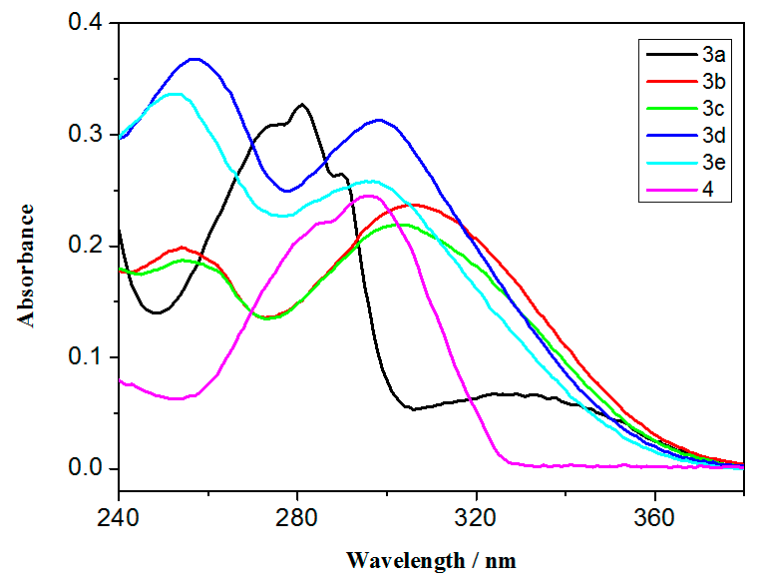

Figure 1. The absorption spectra of compounds 3(a-e), 4.

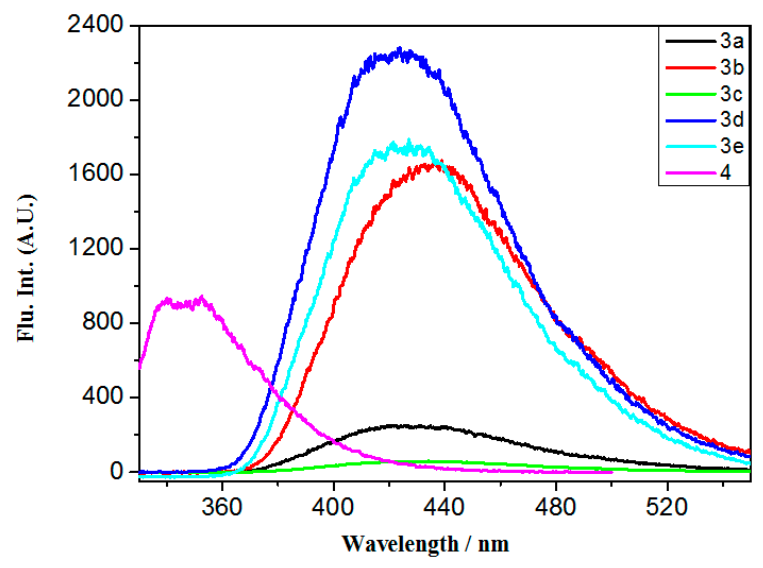

Figure 2. The emission spectra of compounds $3(\mathbf{a}-\mathbf{e}), 4$.

As shown in Table 1, the gradual blue shift of the UV absorbance of 3a-e correlated well with the bulkiness of the corresponding NIT molecule's indole $N$-substitution. Compared with indole $N$-alkyl or phenyl substituted 3b-e (Table 1, entries 3-6), $N$-unsubstituted compound 3a gave a relative weak fluorescence emission (centered at $429 \mathrm{~nm}$ ), possibly because of the intense solvation effect of the bare $\mathrm{N}-\mathrm{H}$ bond in dichloromethane (DCM) $[44,45]$. The highly steric hindered compounds $3 \mathbf{d}-\mathbf{e}$ with a 
bulky $N$-phenyl group gave similar strong emissions as the much less hindered compounds $3 \mathbf{b}$ and 3c, which suggested the small influence of the bulkiness on the indole nitrogen position for the light emission. This effect was also confirmed by the slight red-shift of the emission of $\mathbf{3 b}-\mathbf{c}$ compared with the fluorescence of 3a. Moreover, the gradual increase in the Stokes shift of 3b-e (from 117-127 nm) indicated a much larger difference between the electronic structure of $\mathbf{3 b} \mathbf{b}-\mathbf{e}$ in the ground state and those in the excited state [46]. Notably, the quantum yields of $\mathbf{3 b}$-e were very high; in particular, the quantum yield of $3 \mathbf{e}$ reached up to 0.99 .

Compared with NAT molecule 4, NIT compounds 3a-e presented large, quite interesting Stokes shifts; their electronic structure in the excited state should be conspicuously varied from those in the ground state. In order to determine the NIT molecule's co-planarity in the ground state, the X-ray crystal structure of compounds $3 \mathbf{b}$ and $3 \mathbf{h}$ were studied. As shown in Figure 3, the crystal structure of both $\mathbf{3 b}$ and $\mathbf{3 h}$ exhibited a very large dihedral angle between the indole ring and the triazole ring (see Supplementary Materials). $3 \mathrm{~h}$ in particular exhibited a dihedral angle of up to $72.64^{\circ}$. These compounds' real dihedral angles might be different when dissolved in a solution. However, considering that an intramolecular charge transfer emitter always tends to take a more planar-like configuration at its excited state $[47,48]$, the wide dihedral angles shown in Figure 3 partly verified the large Stokes shift exhibited by NIT molecules in Table 1.

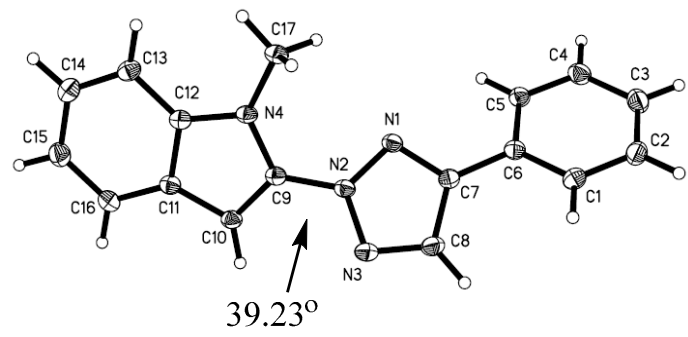

3b

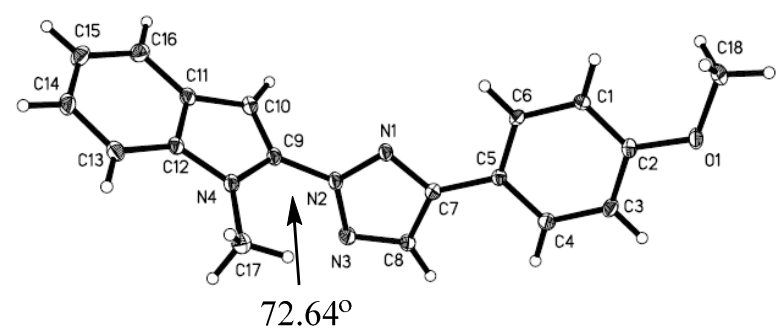

3h

Figure 3. X-ray crystal structures of $3 \mathrm{~b}$ and $3 \mathrm{~h}$.

The effect of triazole substituent $R_{1}$ group (Scheme 2) was then explored. As shown in Table 2, various triazole substitution patterns, such as phenyl (3f-m and $\mathbf{3 o}-\mathbf{p})$, thienyl (3n), and alkyl (3q) groups were studied. It was found their UV absorbance ranged from $294 \mathrm{~nm}$ to $340 \mathrm{~nm}$ (Figure 4), while their fluorescence emission varied from $414 \mathrm{~nm}$ to $467 \mathrm{~nm}$ (Figure 5). The substituents' electronegativity would affect the optical properties of the NITs. For example, a slight $(10-20 \mathrm{~nm})$ red shift of the fluorescence emission peak and the large Stokes shift of 31 was due to its strong donor-acceptor ability [49-51]. The emission peaks of $\mathbf{3 i}$ and $\mathbf{3 o}$ showed obvious red shifts, because of the presence of an additional ethynyl group (3i) and a planar benzotriazole group (3o). Nevertheless, compared with $\mathbf{3 f}-\mathbf{p}$, the UV absorbance and fluorescence emission of alkyl-substituted $\mathbf{3 q}$ and unsubstituted $\mathbf{3 r}$ have obvious blue shifts, due to the absence of a conjugated phenyl or thienyl group [52-54]. The slight red shift of the emission and large Stokes shift of 3m might have resulted from an intramolecular hydrogen bond between the triazole group and the neighboring hydroxyl group. These NIT molecules' quantum 
yields ranged between $0.14-0.84$. 3o and $3 \mathbf{p}$ have relative small quantum yields, while $3 \mathbf{k}$ and $3 \mathbf{r}$ have much larger quantum yields, though their detailed affecting factors are still not clear.

Table 2. Fluorescence emission behavior of compounds with different triazole-substitutions ${ }^{(a)}$.

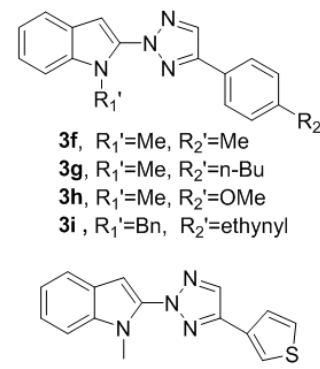

$3 n$<smiles>Cn1c(-n2ncc(-c3cccc(F)c3)n2)cc2ccccc21</smiles>

3j, $\mathrm{R}=\mathrm{OMe}$ $3 \mathrm{k}, \mathrm{R}=\mathrm{Cl}$
$3 \mathrm{l}, \mathrm{R}=\mathrm{F}$

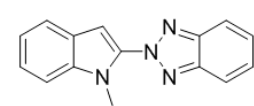

30<smiles>[R]c1ccc2cc(-n3ncc(-c4ccccc4O)n3)n(C)c2c1</smiles>

$3 m$

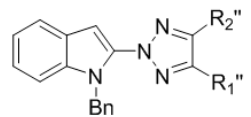

p, $\mathrm{R}_{1}{ }^{\prime \prime}=\mathrm{Ph}, \mathrm{R}_{2} "=\mathrm{Br}$

$3 q, R_{1} "=n-B u, R_{2} "=H$ $3 r, R_{1} "=H, R_{2} "=H$

\begin{tabular}{|c|c|c|c|c|c|}
\hline & Absorption (nm) & Emission $\left(\lambda_{\max }\right)(\mathrm{nm})$ & $\Phi^{(b)}$ & Fluorescence Intensity $\left(\times 10^{3}\right)$ & Stokes Shift \\
\hline $3 f$ & $308(0.302)$ & 427 & 0.71 & 134.75 & 119 \\
\hline $3 g$ & $310(0.337)$ & 434 & 0.46 & 95.14 & 124 \\
\hline $3 h$ & $315(0.306)$ & 432 & 0.44 & 88.16 & 117 \\
\hline $3 \mathbf{i}$ & 315 (0.347) & 443 & 0.62 & 144.93 & 128 \\
\hline $3 \mathbf{j}$ & $315(0.334)$ & 431 & 0.73 & 160.55 & 116 \\
\hline $3 k$ & $310(0.379)$ & 440 & 0.80 & 191.11 & 130 \\
\hline 31 & $306(0.346)$ & 447 & 0.67 & 142.66 & 141 \\
\hline $3 m$ & $310(0.263)$ & 445 & 0.58 & 97.74 & 135 \\
\hline $3 n$ & $305(0.275)$ & 430 & 0.69 & 113.77 & 125 \\
\hline 30 & $340(0.346)$ & 467 & 0.14 & 29.77 & 127 \\
\hline $3 p$ & $295(0.259)$ & 438 & 0.20 & 24.8 & 143 \\
\hline $3 q$ & $295(0.173)$ & 414 & 0.55 & 25.78 & 119 \\
\hline $3 r$ & $294(0.274)$ & 415 & 0.84 & 60.6 & 121 \\
\hline
\end{tabular}

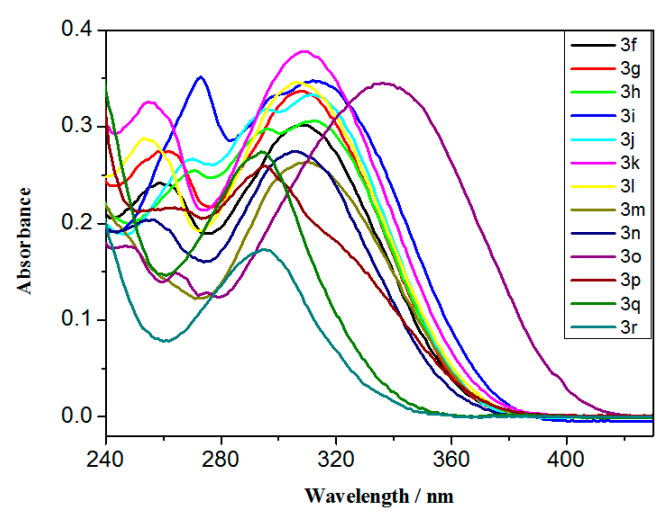

Figure 4. The absorption spectra of compounds $3(\mathbf{f}-\mathbf{r})$.

We then explored the effect of the indole ring substituents. As compared with the fluoro group in 3s, the TsO-group and AcNH-group's low electronegativity as well as their conjugated lone pair improved the donor-acceptor ability of $\mathbf{3 t}$ and especially $\mathbf{3} \mathbf{u}$, thus leading to the red shift of their UV absorbance and fluorescence emission (Figures 6 and 7). The small Stokes shift of $3 \mathbf{u}$ might be due to its better co-planarity in the ground state. As shown in Table 3, 3v has a little red shift in its UV absorbance and fluorescence emission compared to 3a, while its Stokes shift is lower than that of $\mathbf{3 a}$. An additional $N$-benzyl group would reduce the co-planarity of $3 \mathbf{w}$ in the ground state, and then improve its Stokes shift. 


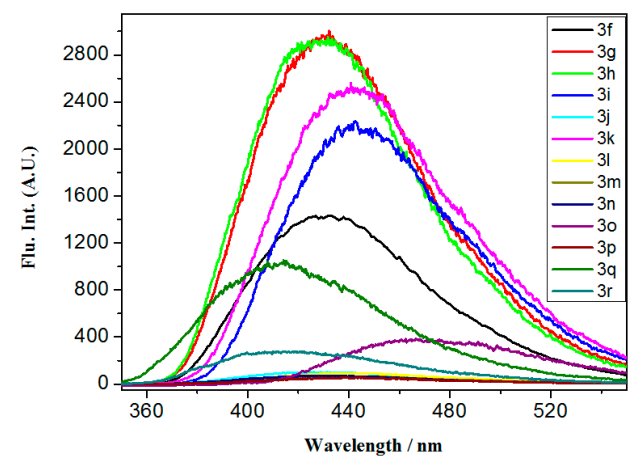

Figure 5. The emission spectra of compounds $3(\mathbf{f}-\mathbf{r})$.

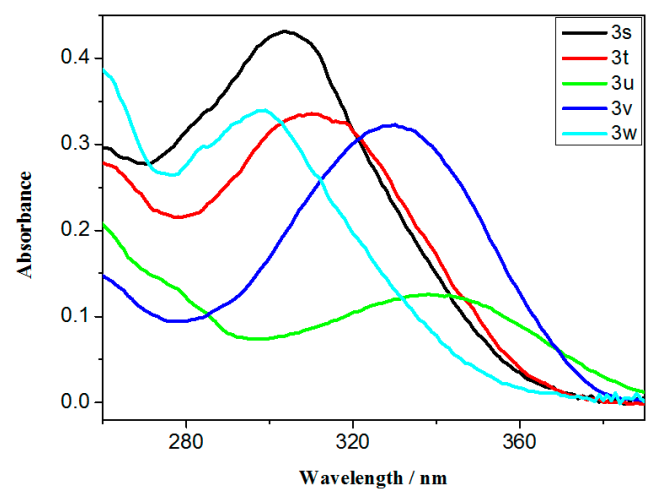

Figure 6. The absorption spectra of compounds $3(\mathbf{s}-\mathbf{w})$.

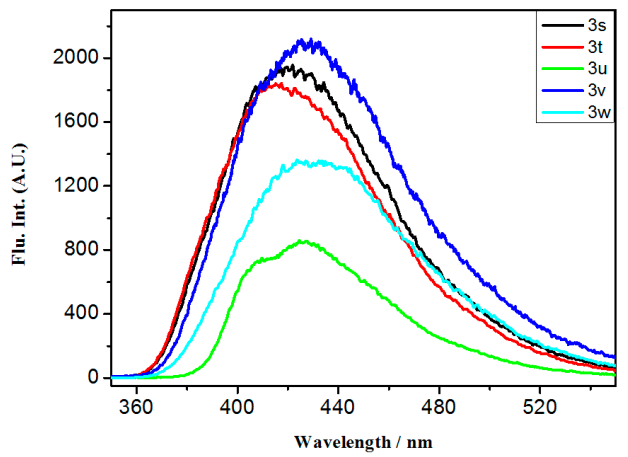

Figure 7. The emission spectra of compounds $3(\mathbf{f}-\mathbf{r})$.

We wondered if the metal complexation of the triazole group and the indole nitrogen atom would improve NIT molecules' co-planarity and affect their optical properties. As shown in Scheme 3, the reactions of NIT molecule $3 \mathbf{v}$ with metal salts $\mathrm{AuCl}_{3}$ and $\mathrm{Pd}(\mathrm{OAc})_{2}$ were tested, in which no desired $\mathrm{Au}(\mathrm{III})$ metal complex was obtained. However, the reaction of $3 \mathbf{v}$ with 1 equivalent of $\mathrm{Pd}(\mathrm{OAc})_{2}$ in $\mathrm{CH}_{2} \mathrm{Cl}_{2}$ at room temperature (r.t.) gave a white solid 5 in $45 \%$ yield (Scheme 3). As confirmed by its X-ray crystal structure shown in Figure 8, the palladium complex was determined to be a metal complex dimer bridged by two acetates, rather like an open book. In complex 5, it was found that the indole ring, the triazole ring, and the palladium atom almost located in a co-facial arrangement. The geometry around $\mathrm{Pd}(\mathrm{II})$ is square planar, composed of two nitrogen atoms from the indole ring and triazole ring, and two oxygen atoms from two bridging acetates. The two NIT ligands are close to parallel in an offset face-to-face stacking mode by a quite small dihedral angle of $0.54^{\circ}$, conforming

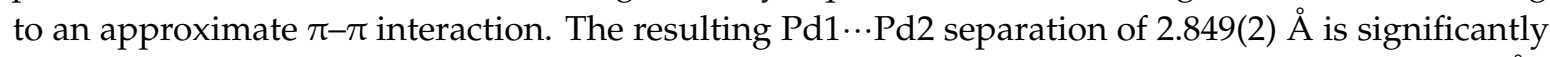
shorter than the sum of the van der Waals radii of palladium (the typical value of which is $1.6 \AA$ ), 
which reveals a Pd $\cdots P d$ interaction [55]. Previous studies on double-carboxylato-bridged dinuclear organometallic $\mathrm{Pd}(\mathrm{II})$ complexes have shown the possibility of cis-trans isomerism depending on the nature of the ligands [56-58]. It has been found that only the trans isomer of complex 5 crystallizes in the asymmetric unit.

Table 3. Fluorescence emission behavior of compounds with different indole-substitutions (a).<smiles></smiles>

3s

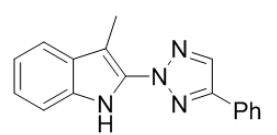

$3 v$<smiles>Oc1ccc2c(c1)cc(-n1ncc(-c3ccccc3)n1)n2Cc1ccccc1</smiles>

$3 t$

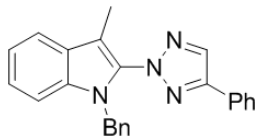

$3 w$

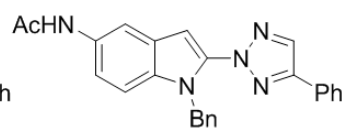

$3 u$

\begin{tabular}{cccccc}
\hline & Absorption (nm) & Emission $(\boldsymbol{\lambda}$ max) $\mathbf{( n m )}$ & $\mathbf{\Phi}^{(\mathbf{b})}$ & Fluorescence Intensity $\left(\times \mathbf{1 0}^{\mathbf{3}}\right)$ & Stokes Shift \\
\hline 3s & $304(0.431)$ & 419 & 0.76 & 165.15 & 115 \\
3t & $310(0.336)$ & 427 & 0.73 & 163.18 & 117 \\
3u & $338(0.126)$ & 427 & 0.85 & 61.62 & 89 \\
3v & $330(0.323)$ & 426 & 0.69 & 143.63 & 96 \\
3w & $299(0.340)$ & 424 & 0.86 & 117.16 & 125 \\
\hline
\end{tabular}

(a) Sample information: $1.0 \times 10^{-5} \mathrm{~mol} \mathrm{~L}^{-1}$ in DCM, excitation at $320 \mathrm{~nm}$ with $2.5 \mathrm{~nm}$ slit. ${ }^{(\mathrm{b})}$ Quantum yields $(\Phi)$ were determined based on $1.0 \times 10^{-5} \mathrm{~mol} \mathrm{~L}^{-1}$ 9,10-Diphenylanthracene in cyclohexane $(\Phi=0.95)$.

Mixture

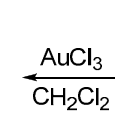

$\frac{\mathrm{Pd}(\mathrm{OAC})_{2}}{\mathrm{CH}_{2} \mathrm{Cl}_{2}}$

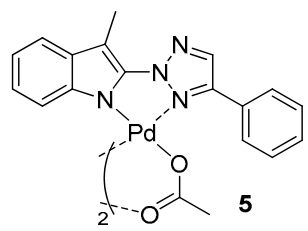

Scheme 3. The reaction of $3 w$ with $\mathrm{AuCl}_{3}$ and $\mathrm{Pd}(\mathrm{OAc})_{2}$.

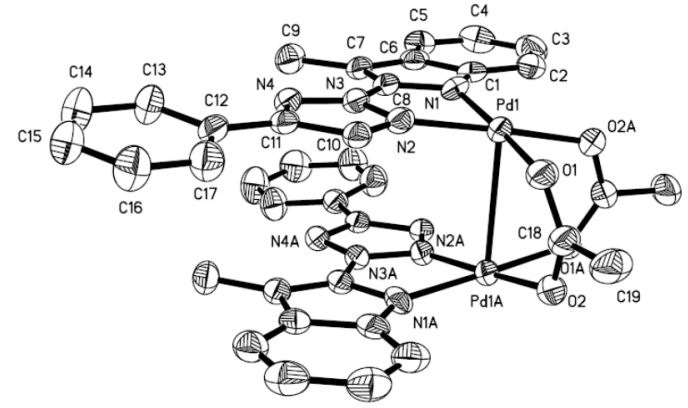

Figure 8. X-ray crystal structure of 5.

However, complex 5 show no fluorescence under UV irradiation, possibly due to the fluorescence quenching by $\mathrm{Pd}^{2+} d-d$ transition.

Finally, the optical properties of two bio-active molecule derivatives $3 \mathbf{x}$ and $3 \mathbf{y}$ were also explored, in which compound $3 \mathbf{x}$ was obtained from the reaction of naturally occurring plant auxin indole-3-acetic acid methyl ester with $N$-1,2,3-phenyl triazole, and $3 \mathbf{y}$ was derived from amino acid tryptophan. As shown in Table 4, compounds $3 \mathbf{x}$ and $3 \mathbf{y}$ absorb strongly at 326-328 nm (Figure 9) and emit vivid blue fluorescence (centered at $418 \mathrm{~nm}$ and $430 \mathrm{~nm}$, Figure 10). In sharp contrast, the intrinsic tryptophan units have negligible absorption in the region with a wavelength longer than $320 \mathrm{~nm}$ and 
consequently no fluorescence under 330-nm excitation, which might enable a reliable detection of target biomolecules tagged with the abovementioned fluorescent tryptophan-triazole conjugate $3 \mathbf{y}$ without the interference from tryptophan components. Considering the unique UV/blue emission of compound $3 y$ and its analogs, it is a potential new kind of UV fluorescence probe for various chemical and biological studies.

Table 4. Fluorescence emission behavior of compounds $3 \mathbf{x}$ and $3 y^{(a)}$.

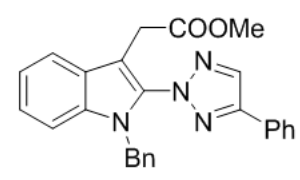

$3 \mathbf{x}$

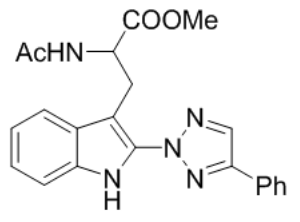

$3 y$

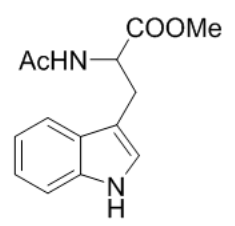

$1 y$

\begin{tabular}{cccccc}
\hline & Absorption (nm) & Emission $(\lambda \mathbf{m a x})(\mathbf{n m})$ & $\boldsymbol{\Phi}^{(\mathbf{b})}$ & Fluorescence Intensity $\left(\times \mathbf{1 0}^{\mathbf{3}}\right)$ & Stokes Shift $^{(28}$ \\
\hline $\mathbf{3 x}$ & $328(0.373)$ & 418 & 0.72 & 173.80 & 90 \\
$\mathbf{3 y}$ & $326(0.409)$ & 430 & 0.60 & 165.49 & 104 \\
\hline
\end{tabular}

(a) Sample information: $1.0 \times 10^{-5} \mathrm{~mol} \mathrm{~L}^{-1}$ in DCM, excitation at $320 \mathrm{~nm}$ with $2.5 \mathrm{~nm}$ slit. ${ }^{\text {(b) }}$ Quantum yields ( $\left.\Phi\right)$ were determined based on $1.0 \times 10^{-5} \mathrm{~mol} \mathrm{~L}^{-1}$ 9,10-Diphenylanthracene in cyclohexane $(\Phi=0.95)$.

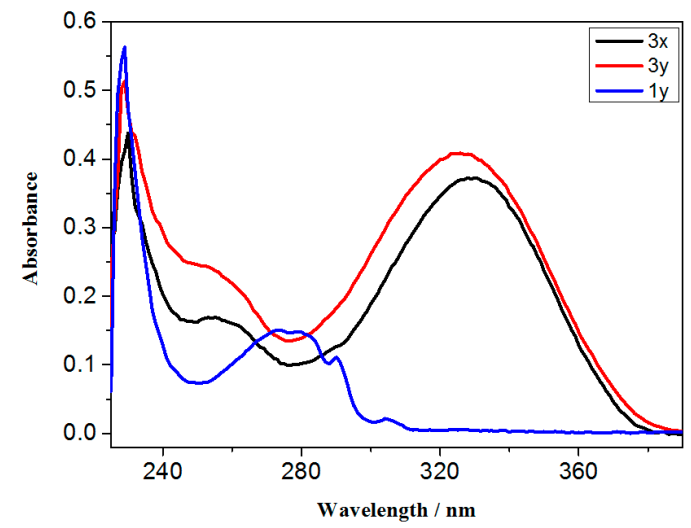

Figure 9. The absorption spectra of compounds $3 \mathbf{x}, 3 \mathbf{y}, \mathbf{1 y}$.

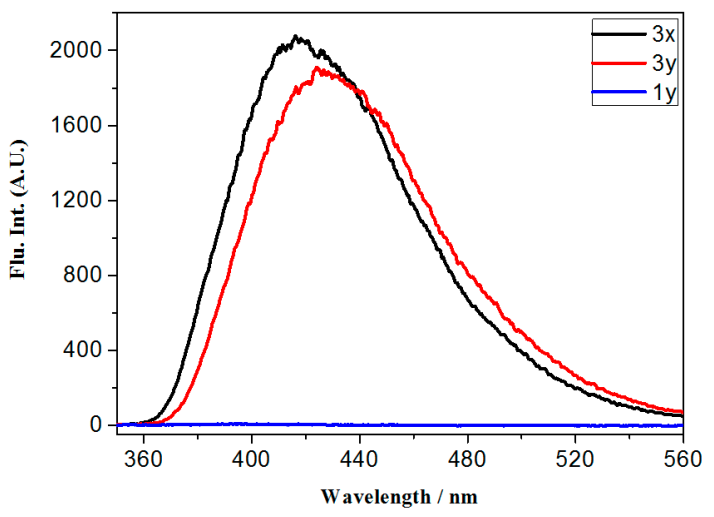

Figure 10. The emission spectra of compounds $3 \mathbf{x}, 3 \mathbf{y}, \mathbf{1 y}$.

\section{Experimental Section}

General procedure for coupling reaction (condition 1) for the synthesis of $\mathbf{3 a} \mathbf{a}-\mathbf{3 u}$ : To a suspension of $\mathrm{N}$-iodo succinimide $(0.3 \mathrm{mmol})$ and $\mathrm{K}_{2} \mathrm{CO}_{3}(0.5 \mathrm{mmol})$ in dry dioxane $(1 \mathrm{~mL})$, was added dropwise a solution of $1 \mathbf{a}(0.2 \mathrm{mmol})$ and $\mathbf{2 a}(0.1 \mathrm{mmol})$ in dioxane $(1 \mathrm{~mL})$ in $5 \mathrm{~min} .30 \mathrm{~min}$ later, the reaction 
mixture was diluted with $20 \mathrm{~mL}$ EtOAc, and was then washed with saturated aqueous $\mathrm{Na}_{2} \mathrm{~S}_{2} \mathrm{O}_{3}$ $(5 \mathrm{~mL})$, brine $(10 \mathrm{~mL})$ and water $(10 \mathrm{~mL})$. The organic phase was dried over anhydrous $\mathrm{Na}_{2} \mathrm{SO}_{4}$, filtered and concentrated in vacuo, purification of the crude product through flash chromatography (petroleum $/$ EtOAc $=50 / 1$ ) afforded $3 \mathbf{a}$ as a white solid in $60 \%$ yield.

2-(4-Phenyl-2H-1,2,3-triazol-2-yl)- $1 H$-indole 3a [41]. Obtained as a white solid in $60 \%$ yield; m.p. 128-130 ${ }^{\circ} \mathrm{C} ;{ }^{1} \mathrm{H}-\mathrm{NMR}\left(400 \mathrm{MHz}, \mathrm{CDCl}_{3}\right) \delta 9.07(\mathrm{br}, 1 \mathrm{H}), 8.07(\mathrm{~s}, 1 \mathrm{H}), 7.89(\mathrm{~d}, J=7.1 \mathrm{~Hz}, 2 \mathrm{H}), 7.66(\mathrm{~d}$, $J=7.8 \mathrm{~Hz}, 1 \mathrm{H}), 7.49(\mathrm{t}, J=7.4 \mathrm{~Hz}, 2 \mathrm{H}), 7.42(\mathrm{dd}, J=7.7,2.8 \mathrm{~Hz}, 2 \mathrm{H}), 7.28-7.22(\mathrm{~m}, 1 \mathrm{H}), 7.21-7.15(\mathrm{~m}$, $1 \mathrm{H}), 6.90(\mathrm{~d}, J=1.4 \mathrm{~Hz}, 1 \mathrm{H}) ;{ }^{13} \mathrm{C}-\mathrm{NMR}\left(101 \mathrm{MHz}, \mathrm{CDCl}_{3}\right) \delta 149.05,134.80,133.54,132.82,129.44,129.16$, $129.06,127.89,126.22,122.86,121.04,120.98,111.20,90.31$.

1-Methyl-2-(4-phenyl-2H-1,2,3-triazol-2-yl)-1H-indole $3 \mathbf{b}$ [41]. Obtained as a white solid in $64 \%$ yield; m.p. 90-91 ${ }^{\circ} \mathrm{C} ;{ }^{1} \mathrm{H}-\mathrm{NMR}\left(400 \mathrm{MHz}, \mathrm{CDCl}_{3}\right) \delta 8.11(\mathrm{~s}, 1 \mathrm{H}), 7.88(\mathrm{~d}, J=7.1 \mathrm{~Hz}, 2 \mathrm{H}), 7.66(\mathrm{~d}, J=7.9 \mathrm{~Hz}$, $1 \mathrm{H}), 7.46(\mathrm{t}, J=7.4 \mathrm{~Hz}, 2 \mathrm{H}), 7.38(\mathrm{dd}, J=12.9,7.8 \mathrm{~Hz}, 2 \mathrm{H}), 7.30(\mathrm{t}, J=7.1 \mathrm{~Hz}, 1 \mathrm{H}), 7.18(\mathrm{t}, J=6.9 \mathrm{~Hz}, 1 \mathrm{H})$, $6.84(\mathrm{~s}, 1 \mathrm{H}), 3.83(\mathrm{~s}, 3 \mathrm{H}) ;{ }^{13} \mathrm{C}-\mathrm{NMR}\left(101 \mathrm{MHz}, \mathrm{CDCl}_{3}\right) \delta 149.08,135.92,135.44,133.02,129.61,129.17$, $129.10,126.27,122.96,121.41,120.71,120.30,109.83,96.27,30.56$.

1-Benzyl-2-(4-phenyl-2H-1,2,3-triazol-2-yl)-1H-indole 3c [41]. Obtained as a white solid in $72 \%$ yield; m.p. $91-92{ }^{\circ} \mathrm{C} ;{ }^{1} \mathrm{H}-\mathrm{NMR}\left(400 \mathrm{MHz}, \mathrm{CDCl}_{3}\right) \delta 8.01(\mathrm{~s}, 1 \mathrm{H}), 7.77(\mathrm{~d}, J=7.2 \mathrm{~Hz}, 2 \mathrm{H}), 7.67(\mathrm{~d}, J=7.6 \mathrm{~Hz}$, $1 \mathrm{H}), 7.39(\mathrm{t}, J=7.3 \mathrm{~Hz}, 2 \mathrm{H}), 7.34(\mathrm{~d}, J=7.1 \mathrm{~Hz}, 1 \mathrm{H}), 7.28(\mathrm{~d}, J=8.1 \mathrm{~Hz}, 1 \mathrm{H}), 7.22(\mathrm{~d}, J=6.9 \mathrm{~Hz}, 1 \mathrm{H})$, 7.20-7.11 (m, 4H), $7.04(\mathrm{~d}, J=7.0 \mathrm{~Hz}, 2 \mathrm{H}), 6.92(\mathrm{~s}, 1 \mathrm{H}), 5.57(\mathrm{~s}, 2 \mathrm{H}) ;{ }^{13} \mathrm{C}-\mathrm{NMR}\left(101 \mathrm{MHz}, \mathrm{CDCl}_{3}\right) \delta$ 149.31, 137.45, 135.77, 135.39, 133.09, 129.60, 129.18, 129.10, 128.70, 127.44, 126.60, 126.44, 126.29, 123.30, $121.57,121.06,110.62,96.96,47.71$.

1-Phenyl-2-(4-phenyl-2H-1,2,3-triazol-2-yl)- $1 H$-indole $3 \mathrm{~d}$. Obtained as a white solid in $57 \%$ yield; m.p. 91-93 ${ }^{\circ} \mathrm{C} ;{ }^{1} \mathrm{H}-\mathrm{NMR}\left(400 \mathrm{MHz}, \mathrm{CDCl}_{3}\right) \delta 8.02(\mathrm{~s}, 1 \mathrm{H}), 7.92-7.78(\mathrm{~m}, 3 \mathrm{H}), 7.43(\mathrm{t}, J=20.5 \mathrm{~Hz}, 11 \mathrm{H}), 7.15(\mathrm{~s}$, $1 \mathrm{H}) ;{ }^{13} \mathrm{C}-\mathrm{NMR}\left(101 \mathrm{MHz}, \mathrm{CDCl}_{3}\right) \delta 149.06,136.91,136.60,135.28,133.03,129.70,129.39,129.08,128.07$, 127.56, 126.27, 123.87, 121.67, 121.63, 111.11, 98.86; IR (neat) 3061, 3028, 1607, 1534, 1461, 1356, 1055, $946,768,723,692,483 \mathrm{~cm}^{-1}$; HRMS(ESI) $\mathrm{m} / z$ calcd. for $\mathrm{C}_{22} \mathrm{H}_{16} \mathrm{~N}_{4},[\mathrm{M}+\mathrm{H}]^{+} 337.1448$, found 337.1444.

1-(4-Methoxyphenyl)-2-(4-phenyl-2H-1,2,3-triazol-2-yl)- $1 H$-indole 3e. Obtained as a white solid in $51 \%$ yield; m.p. $94-96{ }^{\circ} \mathrm{C} ;{ }^{1} \mathrm{H}-\mathrm{NMR}\left(400 \mathrm{MHz}, \mathrm{CDCl}_{3}\right) \delta 7.94(\mathrm{~s}, 1 \mathrm{H}), 7.76-7.71(\mathrm{~m}, 3 \mathrm{H}), 7.45-7.39$ $(\mathrm{m}, 2 \mathrm{H}), 7.37(\mathrm{~d}, J=7.2 \mathrm{~Hz}, 1 \mathrm{H}), 7.27-7.22(\mathrm{~m}, 5 \mathrm{H}), 6.97(\mathrm{~s}, 1 \mathrm{H}), 6.91(\mathrm{~d}, J=8.9 \mathrm{~Hz}, 2 \mathrm{H}), 3.81(\mathrm{~s}, 3 \mathrm{H})$; ${ }^{13} \mathrm{C}-\mathrm{NMR}\left(101 \mathrm{MHz}, \mathrm{CDCl}_{3}\right) \delta 159.12,148.87,136.81,135.28,132.84,129.51,129.22,128.95,128.79$, 126.15, 125.94, 123.56, 121.45, 121.25, 114.38, 110.97, 98.30, 55.56; IR (neat): 3118, 3035, 2924, 1589, 1496, 1454, 1328, 1091, 975, 858, 767, 688, 505 $\mathrm{cm}^{-1}$; HRMS (ESI) calcd. for $\mathrm{C}_{23} \mathrm{H}_{18} \mathrm{~N}_{4} \mathrm{O}[\mathrm{M}+\mathrm{H}]^{+}: 367.1553$; Found: 367.1541.

1-Methyl-2-(4- $p$-tolyl-2H-1,2,3-triazol-2-yl)-1H-indole 3f. Obtained as a white solid in 59\% yield; m.p. 94-96 ${ }^{\circ} \mathrm{C} ;{ }^{1} \mathrm{H}-\mathrm{NMR}\left(400 \mathrm{MHz}, \mathrm{CDCl}_{3}\right) \delta 8.09(\mathrm{~s}, 1 \mathrm{H}), 7.78(\mathrm{~d}, J=8.1 \mathrm{~Hz}, 2 \mathrm{H}), 7.66(\mathrm{~d}, J=7.9 \mathrm{~Hz}, 1 \mathrm{H})$, $7.38(\mathrm{~d}, J=8.2 \mathrm{~Hz}, 1 \mathrm{H}), 7.34-7.25(\mathrm{~m}, 3 \mathrm{H}), 7.19(\mathrm{t}, J=7.0 \mathrm{~Hz}, 1 \mathrm{H}), 6.83(\mathrm{~s}, 1 \mathrm{H}), 3.87(\mathrm{~s}, 3 \mathrm{H}), 2.40(\mathrm{~s}$, $3 \mathrm{H}) ;{ }^{13} \mathrm{C}-\mathrm{NMR}\left(101 \mathrm{MHz}, \mathrm{CDCl}_{3}\right) \delta 149.21,139.17,135.89,135.48,132.85,129.76,126.76,126.15,122.88$, 121.36, 120.65, 109.79, 96.18, 30.87, 21.44; IR (neat) 3056, 3032, 2931, 1559, 1467, 1331, 1145, 966, 772, 701, $536,458 \mathrm{~cm}^{-1}$; HRMS(ESI) $m / z$ calcd. for $\mathrm{C}_{18} \mathrm{H}_{16} \mathrm{~N}_{4},[\mathrm{M}+\mathrm{H}]^{+} 289.1448$, found 289.1439.

2-(4-(4-Butylphenyl)-2H-1,2,3-triazol-2-yl)-1-methyl- $1 H$-indole $3 g$. Obtained as a white solid in $52 \%$ yield; m.p. 87-89 ${ }^{\circ} \mathrm{C}$; ${ }^{1} \mathrm{H}-\mathrm{NMR}\left(400 \mathrm{MHz}, \mathrm{CDCl}_{3}\right) \delta 8.07(\mathrm{~s}, 1 \mathrm{H}), 7.78(\mathrm{~d}, J=8.1 \mathrm{~Hz}, 2 \mathrm{H}), 7.65(\mathrm{~d}$, $J=7.9 \mathrm{~Hz}, 1 \mathrm{H}), 7.35(\mathrm{~d}, J=8.1 \mathrm{~Hz}, 1 \mathrm{H}), 7.27(\mathrm{t}, J=7.8 \mathrm{~Hz}, 3 \mathrm{H}), 7.17(\mathrm{t}, J=7.4 \mathrm{~Hz}, 1 \mathrm{H}), 6.83(\mathrm{~s}, 1 \mathrm{H})$, $3.85(\mathrm{~s}, 3 \mathrm{H}), 2.69-2.60(\mathrm{~m}, 2 \mathrm{H}), 1.69-1.56(\mathrm{~m}, 2 \mathrm{H}), 1.43-1.30(\mathrm{~m}, 2 \mathrm{H}), 0.93(\mathrm{t}, J=7.3 \mathrm{~Hz}, 3 \mathrm{H}) ;{ }^{13} \mathrm{C}-\mathrm{NMR}$ $\left(101 \mathrm{MHz}, \mathrm{CDCl}_{3}\right) \delta 149.48,144.22,135.94,135.54,132.88,129.15,127.13,126.20,122.91,121.38,120.68$, 109.81, 96.18, 35.58, 33.59, 30.89, 22.42, 14.06; IR (neat) 3059, 3028, 1594, 1523, 1336, 988, 956, 845, 747, 526, $412 \mathrm{~cm}^{-1}$; HRMS(ESI) $m / z$ calcd. for $\mathrm{C}_{21} \mathrm{H}_{22} \mathrm{~N}_{4}$, [M + H] 331.1917, found 331.1909.

2-(4-(4-Methoxyphenyl)-2H-1,2,3-triazol-2-yl)-1-methyl- $1 H$-indole $3 \mathrm{~h}$. Obtained as a white solid in $60 \%$ yield; m.p. $92-93{ }^{\circ} \mathrm{C} ;{ }^{1} \mathrm{H}-\mathrm{NMR}\left(400 \mathrm{MHz}, \mathrm{CDCl}_{3}\right) \delta 8.05(\mathrm{~s}, 1 \mathrm{H}), 7.82(\mathrm{~d}, J=8.8 \mathrm{~Hz}, 2 \mathrm{H}), 7.66(\mathrm{~d}$, 
$J=7.9 \mathrm{~Hz}, 1 \mathrm{H}), 7.38(\mathrm{~d}, J=8.2 \mathrm{~Hz}, 1 \mathrm{H}), 7.31(\mathrm{t}, J=7.6 \mathrm{~Hz}, 1 \mathrm{H}), 7.18(\mathrm{t}, J=7.4 \mathrm{~Hz}, 1 \mathrm{H}), 6.99(\mathrm{~d}, J=8.8 \mathrm{~Hz}$, 2H), $6.83(\mathrm{~s}, 1 \mathrm{H}), 3.87(\mathrm{~s}, 3 \mathrm{H}), 3.84(\mathrm{~s}, 3 \mathrm{H}) ;{ }^{13} \mathrm{C}-\mathrm{NMR}\left(101 \mathrm{MHz}, \mathrm{CDCl}_{3}\right) \delta 160.26,149.09,135.86,135.49$, 132.51, 127.57, 126.12, 122.82, 122.18, 121.30, 120.60, 114.44, 109.50, 95.96, 55.37, 30.82; IR (neat) 3061, $2986,1628,1495,1441,1276,1063,987,823,715,681,512 \mathrm{~cm}^{-1}$; HRMS(ESI) $m / z$ calcd. for $\mathrm{C}_{18} \mathrm{H}_{16} \mathrm{~N}_{4} \mathrm{O}$, $[\mathrm{M}+\mathrm{H}]^{+}$305.1397, found 305.1387.

1-Benzyl-2-(4-(4-ethynylphenyl)-2H-1,2,3-triazol-2-yl)-1H-indole 3i. Obtained as a white solid in 53\% yield; m.p. 108-110 ${ }^{\circ} \mathrm{C} ;{ }^{1} \mathrm{H}-\mathrm{NMR}\left(400 \mathrm{MHz}, \mathrm{CDCl}_{3}\right) \delta 8.06(\mathrm{~s}, 1 \mathrm{H}), 7.75(\mathrm{~d}, J=8.0 \mathrm{~Hz}, 2 \mathrm{H}), 7.69(\mathrm{~d}$, $J=7.7 \mathrm{~Hz}, 1 \mathrm{H}), 7.55(\mathrm{~d}, J=8.0 \mathrm{~Hz}, 2 \mathrm{H}), 7.32(\mathrm{~d}, J=8.1 \mathrm{~Hz}, 1 \mathrm{H}), 7.26-7.15(\mathrm{~m}, 5 \mathrm{H}), 7.05(\mathrm{~d}, J=7.2 \mathrm{~Hz}$, 2H), $6.93(\mathrm{~s}, 1 \mathrm{H}), 5.60(\mathrm{~s}, 2 \mathrm{H}), 3.15(\mathrm{~s}, 1 \mathrm{H}) ;{ }^{13} \mathrm{C}-\mathrm{NMR}\left(101 \mathrm{MHz}^{\left.-\mathrm{CDCl}_{3}\right)} \delta 148.36,137.30,135.74,135.14\right.$, 133.11, 132.76, 129.83, 128.62, 127.38, 126.45, 126.30, 126.02, 123.31, 122.74, 121.51, 121.01, 110.51, 97.00, 83.26, 78.53, 47.67; IR (neat) 3356, 3069, 3027, 2109, 1612, 1574, 1452, 1326, 956, 811, 707, 652, $485 \mathrm{~cm}^{-1}$; HRMS(ESI) $m / z$ calcd. for $\mathrm{C}_{25} \mathrm{H}_{18} \mathrm{~N}_{4}$, [M + H] $]^{+} 375.1604$, found 375.1593 .

2-(4-(3-Methoxyphenyl)-2H-1,2,3-triazol-2-yl)-1-methyl-1H-indole 3j. Obtained as a white solid in 58\% yield; m.p. 102-104 ${ }^{\circ} \mathrm{C} ;{ }^{1} \mathrm{H}-\mathrm{NMR}\left(400 \mathrm{MHz}, \mathrm{CDCl}_{3}\right) \delta 8.05(\mathrm{~s}, 1 \mathrm{H}), 7.82(\mathrm{~d}, J=8.3 \mathrm{~Hz}, 2 \mathrm{H}), 7.66(\mathrm{~d}$, $J=7.8 \mathrm{~Hz}, 1 \mathrm{H}), 7.37(\mathrm{~d}, J=8.1 \mathrm{~Hz}, 1 \mathrm{H}), 7.30(\mathrm{t}, J=7.5 \mathrm{~Hz}, 1 \mathrm{H}), 7.18(\mathrm{t}, J=7.3 \mathrm{~Hz}, 1 \mathrm{H}), 6.99(\mathrm{~d}, J=8.3 \mathrm{~Hz}$, 2H), $6.83(\mathrm{~s}, 1 \mathrm{H}), 3.87(\mathrm{~s}, 3 \mathrm{H}), 3.84(\mathrm{~s}, 3 \mathrm{H}) ;{ }^{13} \mathrm{C}-\mathrm{NMR}\left(101 \mathrm{MHz}, \mathrm{CDCl}_{3}\right) \delta 160.33,149.09,135.85,135.48$, 132.51, 127.56, 126.11, 122.82, 122.18, 121.30, 120.60, 114.20, 109.73, 96.08, 55.37, 30.70; IR (neat) 3059, $2923,1619,1486,1442,1253,1072,986,836 \mathrm{~cm}^{-1}$; HRMS(ESI) $\mathrm{m} / z$ calcd. for $\mathrm{C}_{18} \mathrm{H}_{16} \mathrm{~N}_{4} \mathrm{O},[\mathrm{M}+\mathrm{H}]^{+}$ 305.1397, found 305.1390.

2-(4-(3-Chlorophenyl)-2H-1,2,3-triazol-2-yl)-1-methyl-1H-indole 3k. Obtained as a white solid in 65\% yield; m.p. 88-90 ${ }^{\circ} \mathrm{C} ;{ }^{1} \mathrm{H}-\mathrm{NMR}\left(400 \mathrm{MHz}, \mathrm{CDCl}_{3}\right) \delta 8.04(\mathrm{~s}, 1 \mathrm{H}), 7.86(\mathrm{~s}, 1 \mathrm{H}), 7.69(\mathrm{td}, J=4.0,1.5 \mathrm{~Hz}, 1 \mathrm{H})$, $7.64(\mathrm{~d}, J=7.9 \mathrm{~Hz}, 1 \mathrm{H}), 7.36-7.26(\mathrm{~m}, 4 \mathrm{H}), 7.17(\mathrm{t}, J=6.7 \mathrm{~Hz}, 1 \mathrm{H}), 6.82(\mathrm{~s}, 1 \mathrm{H}), 3.82(\mathrm{~s}, 3 \mathrm{H}) ;{ }^{13} \mathrm{C}-\mathrm{NMR}$ $\left(101 \mathrm{MHz}, \mathrm{CDCl}_{3}\right) \delta 147.79,135.83,135.11,134.93,132.89,131.24,130.21,128.96,126.14,125.93,124.17$, 122.94, 121.30, 120.66, 109.73, 96.19, 30.81; IR (neat) 3062, 3016, 1703, 1561, 1467, 1352, 1266, 1056, 956, 796, 737, $628 \mathrm{~cm}^{-1}$; HRMS(ESI) $\mathrm{m} / z$ calcd. for $\mathrm{C}_{17} \mathrm{H}_{13} \mathrm{ClN}_{4}$, $[\mathrm{M}+\mathrm{H}]^{+} 309.0902$, found 309.0893.

2-(4-(3-Fluorophenyl)-2H-1,2,3-triazol-2-yl)-1-methyl-1H-indole 31. Obtained as a white solid in $62 \%$ yield; m.p. 80-82 ${ }^{\circ} \mathrm{C} ;{ }^{1} \mathrm{H}-\mathrm{NMR}\left(400 \mathrm{MHz}, \mathrm{CDCl}_{3}\right) \delta 8.11(\mathrm{~s}, 1 \mathrm{H}), 7.66(\mathrm{t}, J=7.7 \mathrm{~Hz}, 2 \mathrm{H}), 7.61(\mathrm{dd}, J=9.6$, $2.0 \mathrm{~Hz}, 1 \mathrm{H}), 7.46-7.36(\mathrm{~m}, 2 \mathrm{H}), 7.32(\mathrm{t}, J=7.6 \mathrm{~Hz}, 1 \mathrm{H}), 7.19(\mathrm{t}, J=7.4 \mathrm{~Hz}, 1 \mathrm{H}), 7.09(\mathrm{td}, J=8.4,2.5$ $\mathrm{Hz}, 1 \mathrm{H}), 6.85(\mathrm{~s}, 1 \mathrm{H}), 3.88(\mathrm{~s}, 3 \mathrm{H}) ;{ }^{13} \mathrm{C}-\mathrm{NMR}\left(101 \mathrm{MHz}, \mathrm{CDCl}_{3}\right) \delta 164.45,162.00,148.14,135.92,135.21$, 133.03, $131.70(\mathrm{~d}, J=8.4 \mathrm{~Hz}), 130.67(\mathrm{~d}, J=8.3 \mathrm{~Hz}), 126.03,123.03,121.85(\mathrm{~d}, J=2.4 \mathrm{~Hz}), 121.40,120.73$, $116.96(\mathrm{~d}, J=21.3 \mathrm{~Hz}), 113.16(\mathrm{~d}, J=23.0 \mathrm{~Hz}), 109.79,96.35,30.87$; IR (neat) 3059, 3036, 1601, 1553, $1492,1352,1231,1142,956,843,737,628,503 \mathrm{~cm}^{-1}$; HRMS(ESI) $m / z$ calcd. for $\mathrm{C}_{17} \mathrm{H}_{13} \mathrm{FN}_{4},[\mathrm{M}+\mathrm{H}]^{+}$ 293.1197, found 293.1191.

2-(2-(1-Methyl-1H-indol-2-yl)-2H-1,2,3-triazol-4-yl)phenol 3m. Obtained as a white solid in $47 \%$ yield;

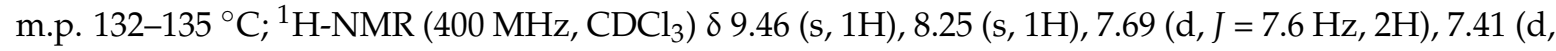
$J=8.2 \mathrm{~Hz}, 1 \mathrm{H}), 7.38-7.30(\mathrm{~m}, 2 \mathrm{H}), 7.22(\mathrm{t}, J=7.4 \mathrm{~Hz}, 1 \mathrm{H}), 7.11(\mathrm{~d}, J=8.2 \mathrm{~Hz}, 1 \mathrm{H}), 7.02(\mathrm{t}, J=7.5 \mathrm{~Hz}, 1 \mathrm{H})$, $6.85(\mathrm{~s}, 1 \mathrm{H}), 3.86(\mathrm{~s}, 3 \mathrm{H}) ;{ }^{1} \mathrm{H}-\mathrm{NMR}\left(400 \mathrm{MHz}, \mathrm{CDCl}_{3}\right): \delta 9.47(\mathrm{~s}, 1 \mathrm{H}), 8.26(\mathrm{~s}, 1 \mathrm{H}), 7.69(\mathrm{~d}, J=8.0 \mathrm{~Hz}$, $2 \mathrm{H}), 7.42(\mathrm{~d}, J=8.2 \mathrm{~Hz}, 1 \mathrm{H}), 7.38-7.33(\mathrm{~m}, 2 \mathrm{H}), 7.23(\mathrm{t}, J=7.4 \mathrm{~Hz}, 1 \mathrm{H}), 7.11(\mathrm{~d}, J=8.3 \mathrm{~Hz}, 1 \mathrm{H}), 7.03(\mathrm{t}$, $J=7.4 \mathrm{~Hz}, 1 \mathrm{H}) 6.85(\mathrm{~s}, 1 \mathrm{H}), 3.87(\mathrm{~s}, 3 \mathrm{H}) ;{ }^{13} \mathrm{C}-\mathrm{NMR}\left(101 \mathrm{MHz}, \mathrm{CDCl}_{3}\right) \delta 155.42,154.18,135.86,132.78$, 130.95, 126.66, 123.34, 121.43, 120.92, 120.08, 117.63, 109.86, 96.80, 88.55, 86.71, 30.82; IR (neat) 3108, 3049, 2986, 1663, 1564, 1474, 1366, 996, 757, 727, $691 \mathrm{~cm}^{-1}$; HRMS(ESI) $m / z$ calcd. for $\mathrm{C}_{17} \mathrm{H}_{14} \mathrm{~N}_{4} \mathrm{O}$, $[\mathrm{M}+\mathrm{H}]^{+}$291.1240, found 291.1236.

1-Methyl-2-(4-(thiophen-3-yl)-2H-1,2,3-triazol-2-yl)-1H-indole 3n. Obtained as a white solid in 51\% yield; m.p. $92-94{ }^{\circ} \mathrm{C} ;{ }^{1} \mathrm{H}-\mathrm{NMR}\left(400 \mathrm{MHz}, \mathrm{CDCl}_{3}\right) \delta 7.97(\mathrm{~s}, 1 \mathrm{H}), 7.70(\mathrm{~d}, J=2.0 \mathrm{~Hz}, 1 \mathrm{H}), 7.64(\mathrm{~d}$, $J=7.9 \mathrm{~Hz}, 1 \mathrm{H}), 7.51(\mathrm{~d}, J=4.8 \mathrm{~Hz}, 1 \mathrm{H}), 7.39-7.36(\mathrm{~m}, 1 \mathrm{H}), 7.34(\mathrm{~d}, J=8.2 \mathrm{~Hz}, 1 \mathrm{H}), 7.28(\mathrm{t}, J=7.5 \mathrm{~Hz}$, 1H), $7.17(\mathrm{t}, J=7.3 \mathrm{~Hz}, 1 \mathrm{H}), 6.82(\mathrm{~s}, 1 \mathrm{H}), 3.82(\mathrm{~s}, 3 \mathrm{H}) ;{ }^{13} \mathrm{C}-\mathrm{NMR}\left(101 \mathrm{MHz}, \mathrm{CDCl}_{3}\right) \delta 145.31,135.82$, $135.26,133.09,130.85,126.73,126.03,125.91,122.87,122.61,121.30,120.61,109.72,96.24,30.44$; IR (neat) 
3033, 2932, 1586, 1454, 1336, 1189, 965, 873, 769, 715; HRMS(ESI) $\mathrm{m} / z$ calcd. for $\mathrm{C}_{15} \mathrm{H}_{12} \mathrm{~N}_{4} \mathrm{~S},[\mathrm{M}+\mathrm{H}]^{+}$ 281.0855, found 281.0847 .

2-(1-Methyl-1H-indol-2-yl)-2H-benzo[d][1,2,3]triazole 3o. Obtained as a white solid in $54 \%$ yield; m.p. 137-139 ${ }^{\circ} \mathrm{C} ;{ }^{1} \mathrm{H}-\mathrm{NMR}\left(400 \mathrm{MHz}, \mathrm{CDCl}_{3}\right) \delta 7.95(\mathrm{dd}, J=6.6,3.1 \mathrm{~Hz}, 2 \mathrm{H}), 7.70(\mathrm{~d}, J=7.9 \mathrm{~Hz}, 1 \mathrm{H})$, 7.50-7.38 (m, 3H), $7.34(\mathrm{t}, J=7.5 \mathrm{~Hz}, 1 \mathrm{H}), 7.21(\mathrm{t}, J=7.1 \mathrm{~Hz}, 1 \mathrm{H}), 7.06(\mathrm{~s}, 1 \mathrm{H}), 4.00(\mathrm{~s}, 3 \mathrm{H}) ;{ }^{13} \mathrm{C}-\mathrm{NMR}$ $\left(101 \mathrm{MHz}, \mathrm{CDCl}_{3}\right) \delta 144.93,136.51,135.80,127.48,126.06,123.41,121.59,120.89,118.31,109.79,97.65$, 31.44; IR (neat) 3052, 3028, 1568, 1452, 1346, 1271, 1059, 956, 771, 732, $692 \mathrm{~cm}^{-1}$; HRMS(ESI) $\mathrm{m} / \mathrm{z}$ calcd. for $\mathrm{C}_{15} \mathrm{H}_{12} \mathrm{~N}_{4},[\mathrm{M}+\mathrm{H}]^{+}$249.1135, found 249.1124.

1-Benzyl-2-(4-bromo-5-phenyl-2H-1,2,3-triazol-2-yl)- $1 H$-indole $3 p$. Obtained as a white solid in 53\% yield; m.p. $90-91{ }^{\circ} \mathrm{C} ;{ }^{1} \mathrm{H}-\mathrm{NMR}\left(400 \mathrm{MHz}, \mathrm{CDCl}_{3}\right) \delta 7.92(\mathrm{~d}, J=6.5 \mathrm{~Hz}, 2 \mathrm{H}), 7.70(\mathrm{~d}, J=7.8 \mathrm{~Hz}, 1 \mathrm{H})$, $7.50-7.40(\mathrm{~m}, 3 \mathrm{H}), 7.34(\mathrm{~d}, J=8.1 \mathrm{~Hz}, 1 \mathrm{H}), 7.28(\mathrm{~d}, J=7.0 \mathrm{~Hz}, 1 \mathrm{H}), 7.24-7.17(\mathrm{~m}, 4 \mathrm{H}), 7.05(\mathrm{~d}, J=6.6 \mathrm{~Hz}$, 2H), $6.93(\mathrm{~s}, 1 \mathrm{H}), 5.61(\mathrm{~s}, 2 \mathrm{H}) ;{ }^{13} \mathrm{C}-\mathrm{NMR}\left(101 \mathrm{MHz}, \mathrm{CDCl}_{3}\right) \delta$ 146.99, 137.07, 135.75, 134.54, 129.39, 128.71, 128.64, 128.32, 127.50, 127.45, 126.77, 126.51, 126.11, 123.52, 121.62, 121.08, 110.51, 97.33, 47.65; IR (neat) 3059, 3030, 2924, 1558, 1454, 1328, 1161, 1010, 956, 727, 694 $\mathrm{cm}^{-1}$; HRMS (ESI) Calcd. for $\mathrm{C}_{23} \mathrm{H}_{18} \mathrm{BrN}_{4}[\mathrm{M}+\mathrm{H}]^{+}$: 429.07094; Found: 429.06996.

1-Benzyl-2-(4-butyl-2H-1,2,3-triazol-2-yl)-1H-indole 3q [41]. Obtained as a colorless oil in $60 \%$ yield; ${ }^{1} \mathrm{H}-\mathrm{NMR}\left(400 \mathrm{MHz}, \mathrm{CDCl}_{3}\right) \delta 7.59(\mathrm{~d}, J=7.7 \mathrm{~Hz}, 1 \mathrm{H}), 7.51(\mathrm{~s}, 1 \mathrm{H}), 7.22(\mathrm{~d}, J=8.0 \mathrm{~Hz}, 1 \mathrm{H}), 7.18-7.05(\mathrm{~m}$, $5 \mathrm{H}), 6.94(\mathrm{~d}, J=6.9 \mathrm{~Hz}, 2 \mathrm{H}), 6.74(\mathrm{~s}, 1 \mathrm{H}), 5.46(\mathrm{~s}, 2 \mathrm{H}), 2.66(\mathrm{t}, J=7.7 \mathrm{~Hz}, 2 \mathrm{H}), 1.83-1.46(\mathrm{~m}, 2 \mathrm{H}), 1.36-1.20$ $(\mathrm{m}, 2 \mathrm{H}), 0.85(\mathrm{t}, J=7.3 \mathrm{~Hz}, 3 \mathrm{H}) ;{ }^{13} \mathrm{C}-\mathrm{NMR}\left(101 \mathrm{MHz}, \mathrm{CDCl}_{3}\right) \delta 150.56,137.32,135.46,134.87,128.54$, $127.28,126.49,126.38,122.96,121.37,120.77,110.44,96.66,47.42,31.09,25.14,22.15,13.75$.

1-Benzyl-2-(2H-1,2,3-triazol-2-yl)-1H-indole $3 \mathbf{r}$ [41]. Obtained as a white solid in $95 \%$ yield; m.p. 95-98 ${ }^{\circ} \mathrm{C} ;{ }^{1} \mathrm{H}-\mathrm{NMR}\left(400 \mathrm{MHz}, \mathrm{CDCl}_{3}\right) \delta 7.76(\mathrm{~s}, 2 \mathrm{H}), 7.61(\mathrm{~d}, J=7.7 \mathrm{~Hz}, 1 \mathrm{H}), 7.22(\mathrm{~d}, J=8.2 \mathrm{~Hz}, 1 \mathrm{H})$, 7.17-7.07 (m, 5H), $6.94(\mathrm{~d}, J=6.7 \mathrm{~Hz}, 2 \mathrm{H}), 6.80(\mathrm{~s}, 1 \mathrm{H}), 5.45(\mathrm{~s}, 2 \mathrm{H}) ;{ }^{13} \mathrm{C}-\mathrm{NMR}\left(101 \mathrm{MHz}, \mathrm{CDCl}_{3}\right) \delta$ 137.16, 135.97, 135.51, 135.14, 128.69, 127.37, 126.43, 126.25, 123.24, 121.58, 120.92, 110.43, 97.20, 47.57.

5-Fluoro-2-(4-phenyl-2H-1,2,3-triazol-2-yl)-1H-indole 3s [41]. Obtained as a white solid in $78 \%$ yield; m.p. $116-119{ }^{\circ} \mathrm{C} ;{ }^{1} \mathrm{H}-\mathrm{NMR}\left(400 \mathrm{MHz}, \mathrm{CDCl}_{3}\right) \delta 8.08(\mathrm{~s}, 1 \mathrm{H}), 7.81(\mathrm{~d}, J=7.0 \mathrm{~Hz}, 2 \mathrm{H}), 7.48-7.38(\mathrm{~m}, 3 \mathrm{H})$, $7.33(\mathrm{dd}, J=9.2,2.4 \mathrm{~Hz}, 1 \mathrm{H}), 7.26-7.18(\mathrm{~m}, 4 \mathrm{H}), 7.06(\mathrm{~d}, J=6.6 \mathrm{~Hz}, 2 \mathrm{H}), 6.98(\mathrm{td}, J=9.1,2.5 \mathrm{~Hz}, 1 \mathrm{H})$, $6.89(\mathrm{~s}, 1 \mathrm{H}), 5.61(\mathrm{~s}, 2 \mathrm{H}) ;{ }^{13} \mathrm{C}-\mathrm{NMR}\left(101 \mathrm{MHz}, \mathrm{CDCl}_{3}\right) \delta 158.46(\mathrm{~d}, J=235.9 \mathrm{~Hz}), 149.40,137.06,136.43$, $133.17,132.19,129.38,129.19,129.04,128.69,127.50,126.70,126.59,126.45,126.22,111.84,111.53(\mathrm{~d}$, $J=9.5 \mathrm{~Hz}), 111.39,106.24(\mathrm{~d}, J=23.6 \mathrm{~Hz}), 96.72(\mathrm{~d}, J=4.5 \mathrm{~Hz}), 96.69,47.85$.

1-Benzyl-2-(4-phenyl-2H-1,2,3-triazol-2-yl)-1H-indol-5-yl-4-methylbenzenesulfonate 3t [41]. Obtained as a white solid in $83 \%$ yield; m.p. ${ }^{169-171}{ }^{\circ} \mathrm{C} ;{ }^{1} \mathrm{H}-\mathrm{NMR}\left(400 \mathrm{MHz}, \mathrm{CDCl}_{3}\right) \delta 8.07(\mathrm{~s}, 1 \mathrm{H}), 7.80(\mathrm{~d}$, $J=6.9 \mathrm{~Hz}, 2 \mathrm{H}), 7.70(\mathrm{~d}, J=8.3 \mathrm{~Hz}, 2 \mathrm{H}), 7.48-7.36(\mathrm{~m}, 3 \mathrm{H}), 7.28(\mathrm{~d}, J=8.1 \mathrm{~Hz}, 2 \mathrm{H}), 7.25-7.18(\mathrm{~m}, 5 \mathrm{H})$, $7.04(\mathrm{~d}, J=6.3 \mathrm{~Hz}, 2 \mathrm{H}), 6.89(\mathrm{dd}, J=8.9,2.3 \mathrm{~Hz}, 1 \mathrm{H}), 6.84(\mathrm{~s}, 1 \mathrm{H}), 5.58(\mathrm{~s}, 2 \mathrm{H}), 2.43(\mathrm{~s}, 3 \mathrm{H}) ;{ }^{13} \mathrm{C}-\mathrm{NMR}$ $\left(101 \mathrm{MHz}, \mathrm{CDCl}_{3}\right) \delta 149.50,145.15,144.25,136.78,136.42,133.98,133.28,132.48,129.71,129.26,129.06$, $128.70,128.64,127.58,126.50,126.22,123.62,118.06,114.65,111.24,97.07,47.96,21.76$.

$\mathrm{N}$-(1-Benzyl-2-(4-phenyl-2H-1,2,3,-triazol-2-yl)-1H-indol-5-yl)acetamide 3u [41]. Obtained as a white solid in 60\% yield; m.p. $128-131{ }^{\circ} \mathrm{C}$; ${ }^{1} \mathrm{H}-\mathrm{NMR}\left(400 \mathrm{MHz}, \mathrm{CDCl}_{3}\right) \delta 8.06(\mathrm{~s}, 1 \mathrm{H}), 7.87(\mathrm{br}, 1 \mathrm{H}), 7.80(\mathrm{~d}$, $J=7.2 \mathrm{~Hz}, 2 \mathrm{H}), 7.51-7.36(\mathrm{~m}, 4 \mathrm{H}), 7.30-7.15(\mathrm{~m}, 5 \mathrm{H}), 7.04(\mathrm{~d}, J=6.8 \mathrm{~Hz}, 2 \mathrm{H}), 6.87(\mathrm{~s}, 1 \mathrm{H}), 5.58(\mathrm{~s}, 2 \mathrm{H})$, $2.17(\mathrm{~s}, 3 \mathrm{H}) ;{ }^{13} \mathrm{C}-\mathrm{NMR}\left(101 \mathrm{MHz}, \mathrm{CDCl}_{3}\right) \delta 168.44,149.27,137.20,135.88,133.04,131.50,129.43,129.13$, $129.02,128.63,127.40,126.46,126.21,117.51,113.23,110.78,96.88,47.77,24.49$.

General procedure for coupling reaction (condition 2) for the synthesis of $3 \mathbf{v}-\mathbf{3 y}$ : To a suspension of $1 \mathbf{v}(0.1 \mathrm{mmol}), 2 \mathbf{a}(0.2 \mathrm{mmol})$ in dry dioxane $(1 \mathrm{~mL})$, was added $0.1 \mathrm{~mL} \mathrm{CHCl}$, and then added dropwise a solution of $N$-iodosuccinimide $(0.3 \mathrm{mmol})$ in dioxane $(1 \mathrm{~mL})$ in $5 \mathrm{~min} .30 \mathrm{~min}$ later, the reaction mixture was diluted with $20 \mathrm{~mL}$ EtOAc, and was washed with saturated aqueous $\mathrm{Na}_{2} \mathrm{~S}_{2} \mathrm{O}_{3}$ $(5 \mathrm{~mL})$, brine $(10 \mathrm{~mL})$ and water $(10 \mathrm{~mL})$. The organic phase was dried over anhydrous $\mathrm{Na}_{2} \mathrm{SO}_{4}$, 
filtered and concentrated in vacuo, purification of the crude product through flash chromatography (petroleum $/ \mathrm{EtOAc}=50 / 1$ as the eluent) afforded $3 \mathbf{v}$ as a white solid.

3-Methyl-2-(4-phenyl-2H-1,2,3,-triazol-2-yl)- $1 H$-indole 3v [41]. Obtained as a white solid in 55\% yield; m.p. $132-134{ }^{\circ} \mathrm{C} ;{ }^{1} \mathrm{H}-\mathrm{NMR}\left(400 \mathrm{MHz}, \mathrm{CDCl}_{3}\right) \delta 8.88(\mathrm{br}, 1 \mathrm{H}), 8.03(\mathrm{~s}, 1 \mathrm{H}), 7.86(\mathrm{~d}, J=5.3 \mathrm{~Hz}, 2 \mathrm{H}), 7.60$ $(\mathrm{d}, J=7.8 \mathrm{~Hz}, 1 \mathrm{H}), 7.45(\mathrm{t}, J=7.4 \mathrm{~Hz}, 2 \mathrm{H}), 7.41-7.35(\mathrm{~m}, 1 \mathrm{H}), 7.31(\mathrm{~d}, J=8.0 \mathrm{~Hz}, 1 \mathrm{H}), 7.23(\mathrm{t}, J=7.0 \mathrm{~Hz}$, $1 \mathrm{H}), 7.17(\mathrm{t}, J=7.0 \mathrm{~Hz}, 1 \mathrm{H}), 2.66(\mathrm{~s}, 3 \mathrm{H}) ;{ }^{13} \mathrm{C}-\mathrm{NMR}\left(101 \mathrm{MHz}, \mathrm{CDCl}_{3}\right) \delta 148.41,132.86,132.15,130.67$, $129.67,129.14,129.05,129.02,126.17,123.07,120.22,119.38,110.88,100.67,9.02$.

1-Benzyl-3-methyl-2-(4-phenyl-2H-1,2,3,-triazol-2-yl)-1H-indole 3w [41]. Obtained as a white solid in 75\% yield; m.p. $78-80{ }^{\circ} \mathrm{C} ;{ }^{1} \mathrm{H}-\mathrm{NMR}\left(400 \mathrm{MHz}, \mathrm{CDCl}_{3}\right) \delta 8.09(\mathrm{~s}, 1 \mathrm{H}), 7.81(\mathrm{~d}, J=7.0 \mathrm{~Hz}, 2 \mathrm{H}), 7.66(\mathrm{~d}$, $J=7.8 \mathrm{~Hz}, 1 \mathrm{H}), 7.43(\mathrm{t}, J=7.3 \mathrm{~Hz}, 2 \mathrm{H}), 7.40-7.34(\mathrm{~m}, 1 \mathrm{H}), 7.30-7.23(\mathrm{~m}, 2 \mathrm{H}), 7.21-7.13(\mathrm{~m}, 4 \mathrm{H}), 7.03(\mathrm{~d}$, $J=6.4 \mathrm{~Hz}, 2 \mathrm{H}), 5.26(\mathrm{~s}, 2 \mathrm{H}), 2.36(\mathrm{~s}, 3 \mathrm{H}) ;{ }^{13} \mathrm{C}-\mathrm{NMR}\left(101 \mathrm{MHz}, \mathrm{CDCl}_{3}\right) \delta 149.24,137.35,134.70,133.01$, $131.66,129.69,129.09,129.07,128.60,127.39,126.90,126.68,126.26,123.67,120.17,119.99,110.33,107.43$, $47.18,8.39$.

Methyl 2-(1-benzyl-2-(4-phenyl-2H-1,2,3,-triazol-2-yl)-1H-indol-3-yl)acetate 3x [41]. Obtained as a white solid in $67 \%$ yield; m.p. $104-106{ }^{\circ} \mathrm{C} ;{ }^{1} \mathrm{H}-\mathrm{NMR}\left(400 \mathrm{MHz}, \mathrm{CDCl}_{3}\right) \delta 8.11(\mathrm{~s}, 1 \mathrm{H}), 7.82(\mathrm{~d}, J=7.0 \mathrm{~Hz}$, $2 \mathrm{H}), 7.69(\mathrm{~d}, J=7.8 \mathrm{~Hz}, 1 \mathrm{H}), 7.45(\mathrm{t}, J=7.3 \mathrm{~Hz}, 2 \mathrm{H}), 7.42-7.37(\mathrm{~m}, 1 \mathrm{H}), 7.33-7.27(\mathrm{~m}, 2 \mathrm{H}), 7.24-7.18(\mathrm{~m}$, $4 \mathrm{H}), 7.06(\mathrm{~d}, J=6.6 \mathrm{~Hz}, 2 \mathrm{H}), 5.42(\mathrm{~s}, 2 \mathrm{H}), 3.91(\mathrm{~s}, 2 \mathrm{H}), 3.63(\mathrm{~s}, 3 \mathrm{H}) ;{ }^{13} \mathrm{C}-\mathrm{NMR}\left(101 \mathrm{MHz}, \mathrm{CDCl}_{3}\right) \delta 171.49$, 149.37, 137.06, 134.65, 133.19, 132.61, 129.49, 129.14, 129.03, 128.61, 127.41, 126.62, 126.23, 123.76, 120.82, $119.90,110.55,103.39,52.09,47.46,29.79$.

(S)-Methyl 2-acetamido-3-(2-(4-phenyl-2H-1,2,3,-triazol-2-yl)-1H-indol-3-yl) propanoate 3y [41]. Obtained as a yellow solid in $62 \%$ yield, $[\alpha]_{D}^{20}=+15.1(c=1.00)$; m.p. $137-140{ }^{\circ} \mathrm{C} ;{ }^{1} \mathrm{H}-\mathrm{NMR}(400$ $\mathrm{MHz}, \mathrm{DMSO}) \delta 12.10(\mathrm{br}, 1 \mathrm{H}), 8.74(\mathrm{~s}, 1 \mathrm{H}), 8.47(\mathrm{~d}, J=7.6 \mathrm{~Hz}, 1 \mathrm{H}), 8.06(\mathrm{~d}, J=7.2 \mathrm{~Hz}, 2 \mathrm{H}), 7.64(\mathrm{~d}$, $J=7.9 \mathrm{~Hz}, 1 \mathrm{H}), 7.56(\mathrm{t}, J=7.5 \mathrm{~Hz}, 2 \mathrm{H}), 7.50-7.42(\mathrm{~m}, 2 \mathrm{H}), 7.20(\mathrm{t}, J=7.5 \mathrm{~Hz}, 1 \mathrm{H}), 7.11(\mathrm{t}, J=7.4 \mathrm{~Hz}, 1 \mathrm{H})$, $4.69(\mathrm{q}, J=7.5 \mathrm{~Hz}, 1 \mathrm{H}), 3.64(\mathrm{dd}, J=13.9,7.4 \mathrm{~Hz}, 1 \mathrm{H}), 3.44(\mathrm{~s}, 3 \mathrm{H}), 3.37(\mathrm{dd}, J=13.9,7.5 \mathrm{~Hz}, 1 \mathrm{H}), 1.77(\mathrm{~s}$, 3H); ${ }^{13} \mathrm{C}-\mathrm{NMR}(101 \mathrm{MHz}$, DMSO) $\delta$ 172.82, 169.65, 148.85, 133.76, 133.60, 131.78, 129.63, 129.58, 128.12, $126.49,125.90,122.93,120.23,119.27,112.15,100.02,53.34,52.10,26.63,22.76$.

Synthetic procedure for compound 5: A solution of $3 \mathbf{v}(0.05 \mathrm{mmol})$ and $\mathrm{Pd}(\mathrm{OAc})_{2}(0.05 \mathrm{mmol})$ in dry $\mathrm{CH}_{2} \mathrm{Cl}_{2}(1 \mathrm{~mL})$ was stirred at room temperature for $2 \mathrm{~h}$. After filtration, slow evaporation of the resulting solution gave a colorless crystal 5 .

Compound 5. Obtained as a yellow solid in $45 \%$ yield, ${ }^{1} \mathrm{H}-\mathrm{NMR}\left(400 \mathrm{MHz}, \mathrm{CDCl}_{3}\right) \delta 7.43-7.38$ $(\mathrm{m}, 1 \mathrm{H}), 7.36(\mathrm{dt}, J=7.8,3.8 \mathrm{~Hz}, 1 \mathrm{H}), 7.01-6.94(\mathrm{~m}, 1 \mathrm{H}), 6.72(\mathrm{~d}, J=8.0 \mathrm{~Hz}, 1 \mathrm{H}), 6.61(\mathrm{ddd}, J=7.9,5.2$, $2.7 \mathrm{~Hz}, 1 \mathrm{H}), 6.57(\mathrm{~s}, 1 \mathrm{H}), 2.38(\mathrm{~s}, 1 \mathrm{H}), 2.30(\mathrm{~s}, 1 \mathrm{H}) ;{ }^{13} \mathrm{C}-\mathrm{NMR}\left(101 \mathrm{MHz}, \mathrm{CDCl}_{3}\right) \delta$ 193.64, 186.49, 180.98, $173.69,161.51,129.30,128.48,126.61,126.02,121.68,119.74,117.96,111.80,98.63,23.79$, , 7.65.4.

\section{Conclusions}

In conclusion, N-2-indolyl-triazoles (NITs) are a novel class of effective UV/blue-light-emitting dyes. Through a comprehensive comparison of the substituted groups on the indole nitrogen atom, the indole group, and the triazole group, a general trend was revealed regarding how to effectively adjust the photoactivity of these compounds. Both emission wavelengths (410-470 nm) and Stokes shifts $(89-143 \mathrm{~nm})$ could be adjusted with various substituted functional groups. We hope that further derivatizations of these NIT molecules would gave highly efficient UV-emitting biocompatible amino acid NIT probes, thus supporting the potential use of these novel dyes in related chemical, material, and biological applications.

Supplementary Materials: A brief experimental details, and spectral data for all new products and NOESY spectra for compound $3 \mathbf{b}, 3 \mathbf{h}$, and $\mathbf{5}$ are available online.

Acknowledgments: Support for this work with the grant from the National Sciences Foundation of China (No. 21472237) is gratefully acknowledged. 
Author Contributions: Zili Chen and Li-Min Fu conceived and designed the experiments; You-Can Zhang performed the experiments; Rui Jin and Luo-Yuan Li analyzed the data.

Conflicts of Interest: The authors declare no conflict of interest.

\section{References}

1. Saha, S.; Stoddart, J.F. Photo-driven molecular devices. Chem. Soc. Rev. 2007, 36, 77-92. [CrossRef] [PubMed]

2. Sakai, N.; Sisson, A.L.; Burgi, T.; Matile, S. Zipper assembly of photoactive rigid-rod naphthalenediimide $\pi$-stack architectures on gold nanoparticles and gold electrodes. J. Am. Chem. Soc. 2007, 129, 15758-15759. [CrossRef] [PubMed]

3. Diaz, D.D.; Cid, J.J.; Vazquez, P.; Torres, T. Strength enhancement of nanostructured organogels through inclusion of phthalocyanine-containing complementary organogelator structures and In Situ cross-linking by click chemistry. Chem. Eur. J. 2008, 14, 9261-9273. [CrossRef] [PubMed]

4. Armstrong, N.R.; Veneman, P.A.; Ratcliff, E.; Placencia, D.; Brumbach, M. Oxide contacts in organic photovoltaics: Characterization and control of near-surface composition in indium-Tin oxide (ITO) electrodes. Acc. Chem. Res. 2009, 42, 1748-1757. [CrossRef] [PubMed]

5. Singhal, K.; Kalkan, A.K. Surface-enhanced Raman scattering captures conformational changes of single photoactive yellow protein molecules under photoexcitation. J. Am. Chem. Soc. 2010, 132, 429-431. [CrossRef] [PubMed]

6. Medintz, I.L.; Uyeda, H.T.; Goldman, E.R.; Mattoussi, H. Quantum dot bioconjugates for imaging, labelling and sensing. Nat. Mater. 2005, 4, 435-446. [CrossRef] [PubMed]

7. Low, P.S.; Henne, W.A.; Doorneweerd, D.D. Discovery and development of folic-acid-based receptor targeting for imaging and therapy of cancer and inflammatory diseases. Acc. Chem. Res. 2008, 41, 120-129. [CrossRef] [PubMed]

8. Takaoka, Y.; Sakamoto, T.; Tsukiji, S.; Narazaki, M.; Matsuda, T.; Tochio, H.; Shirakawa, M.; Hamachi, I. Self-assembling nanoprobes that display off/on 19F nuclear magnetic resonance signals for protein detection and imaging. Nat. Chem. 2009, 1, 557-561. [CrossRef] [PubMed]

9. Jayagopal, A.; Halfpenny, K.C.; Perez, J.W.; Wright, D.W. Hairpin DNA-functionalized gold colloids for the imaging of mRNA in live cells. J. Am. Chem. Soc. 2010, 132, 9789-9796. [CrossRef] [PubMed]

10. Yang, Y.; Seidlits, S.K.; Adams, M.M.; Lynch, V.M.; Schmidt, C.E.; Anslyn, E.V.; Shear, J.B. A highly selective low-background fluorescent imaging agent for nitric oxide. J. Am. Chem. Soc. 2010, 132, 13114-13116. [CrossRef] [PubMed]

11. Gao, M.-X.; Yang, L.; Zheng, Y.; Yang, X.-X.; Zou, H.Y.; Han, J.; Liu, Z.-X.; Li, Y.-F.; Huang, C.-Z. "Click" on alkynylated carbon quantum dots: an efficient surface functionalization for specific biosensing and bioimaging. Chem. Eur. J. 2017, 23, 2171-2178. [CrossRef] [PubMed]

12. Sohn, Y.S.; Goodey, A.; Anslyn, E.V.; McDevitt, J.T.; Shear, J.B.; Neikirk, D.P. A microbead array chemical sensor using capillary-based sample introduction: toward the development of an "electronic tongue". Biosens. Bioelectron. 2005, 21, 303-312. [CrossRef] [PubMed]

13. Khong, S.H.; Sivaramakrishnan, S.; Png, R.Q.; Wong, L.Y.; Chia, P.J.; Chua, L.L.; Ho, P.K.H. General photo-patterning of polyelectrolyte thin films via efficient ionic bis(fluorinated phenyl azide) photo-crosslinkers and their post-deposition Modification. Adv. Funct. Mater. 2007, 17, 2490-2499. [CrossRef]

14. Tian, E.T.; Wang, J.X.; Zheng, Y.M.; Song, Y.L.; Jiang, L.; Zhu, D.B. Colorful humidity sensitive photonic crystal hydrogel. J. Mater. Chem. 2008, 18, 1116-1122. [CrossRef]

15. Ensafi, A.A.; Far, A.K.; Meghdadi, S. Highly selective optical-sensing film for lead (II) determination in water samples. J. Hazard. Mater. 2009, 172, 1069-1075. [CrossRef] [PubMed]

16. Zhao, Y.S.; Zhan, P.; Kim, J.; Sun, C.; Huang, J.X. Patterned growth of vertically aligned organic nanowire waveguide arrays. ACS Nano 2010, 4, 1630-1636. [CrossRef] [PubMed]

17. Sparano, B.A.; Koide, K. Fluorescent sensors for specific RNA: a general paradigm using chemistry and combinatorial biology. J. Am. Chem. Soc. 2007, 129, 4785-4794. [CrossRef] [PubMed]

18. Yan, D.C.; Mohsseni-Ala, J.; Auner, N.; Bolte, M.; Bats, J.W. Molecular optical switches: synthesis, structure, and photoluminescence of spirosila compounds. Chem. Eur. J. 2007, 13, 7204-7214. [CrossRef] [PubMed]

19. Zhang, X.Y.; Matsuo, Y.; Nakamura, E. Light emission of cyclophenacene through energy transfer from neighboring carbazolylphenyl dendrons. Org. Lett. 2008, 10, 4145-4147. [CrossRef] [PubMed] 
20. Dan-Hardi, M.; Serre, C.; Frot, T.; Rozes, L.; Maurin, G.; Sanchez, C.; Ferey, G. A new photoactive crystalline highly porous titanium (IV) dicarboxylate. J. Am. Chem. Soc. 2009, 131, 10857-10859. [CrossRef] [PubMed]

21. Wahab, M.A.; Hussain, H.; He, C. Photoactive perylenediimide-bridged silsesquioxane functionalized periodic mesoporous organosilica thin films (PMO-SBA15): synthesis, self-assembly, and photoluminescent and enhanced mechanical properties. Langmuir 2009, 25, 4743-4750. [CrossRef] [PubMed]

22. Mercs, L.; Albrecht, M. Beyond catalysis: N-heterocyclic carbene complexes as components for medicinal, luminescent, and functional materials applications. Chem. Soc. Rev. 2010, 39, 1903-1912. [CrossRef] [PubMed]

23. Chen, H.; Cheng, N.; Ma, W.; Li, M.; Hu, S.; Gu, L.; Meng, S.; Guo, X. Design of a photoactive hybrid bilayer dielectric for flexible nonvolatile organic memory transistors. ACS Nano 2016, 10, 436-445. [CrossRef] [PubMed]

24. Müller, C.D.; Falcou, A.; Reckefuss, N.; Rojahn, M.; Wiederhirn, V.; Rudati, P.; Frohne, H.; Nuyken, O.; Becker, H.; Meerholz, K. Multi-colour organic light-emitting displays by solution processing. Nature 2003, 421, 829-833. [CrossRef] [PubMed]

25. Kuo, J.S.; Kuyper, C.L.; Allen, P.B.; Fiorini, G.S.; Chiu, D.T. High-power blue/UV light-emitting diodes as excitation sources for sensitive detection. Electrophoresis 2004, 25, 3796-3804. [CrossRef] [PubMed]

26. Liu, X.M.; He, C.B.; Huang, J.C.; Xu, J.M. Highly efficient blue-light-emitting glass-forming molecules based on tetraarylmethane/silane and fluorene: synthesis and thermal, optical, and electrochemical properties. Chem. Mater. 2005, 17, 434-441. [CrossRef]

27. Tsai, M.H.; Hong, Y.H.; Chang, C.H.; Su, H.C.; Wu, C.C.; Matoliukstyte, A.; Simokaitiene, J.; Grigalevicius, S.; Grazulevicius, J.V.; Hsu, C.P. 3-(9-Carbazolyl)carbazoles and 3,6-di(9-carbazolyl)carbazoles as effective host materials for efficient blue organic electrophosphorescence. Adv. Mater. 2007, 19, 862-866. [CrossRef]

28. Li, H.Y.; Batsanov, A.S.; Moss, K.C.; Vaughan, H.L.; Dias, F.B.; Kamtekar, K.T.; Bryce, M.R.; Monkman, A.P. The interplay of conformation and photophysical properties in deep-blue fluorescent oligomers. Chem. Commun. 2010, 46, 4812-4814. [CrossRef] [PubMed]

29. Gaal, M.; List, E.J.W.; Scherf, U. Excimers or emissive on-chain defects? Macromolecules 2003, 36, $4236-4237$. [CrossRef]

30. Zhang, H.C.; Guo, E.Q.; Fang, Y.J.; Ren, P.H.; Yang, W.J. Synthesis and optoelectronic properties of alternating benzofuran/terfluorene copolymer with stable blue emission. J. Polym. Sci. Part. A Polym. Chem. 2009, 47, 5488-5497. [CrossRef]

31. Tonzola, C.J.; Kulkarni, A.P.; Gifford, A.P.; Kaminsky, W.; Jenekhe, S.A. Blue-light-emitting oligoquinolines: synthesis, properties, and high-efficiency blue-light-emitting diodes. Adv. Funct. Mater. 2007, 17, 863-874. [CrossRef]

32. Tong, Q.X.; Lai, S.L.; Chan, M.Y.; Zhou, Y.C.; Kwong, H.L.; Lee, C.S.; Lee, S.T. Highly efficient blue organic light-emitting device based on a nondoped electroluminescent material. Chem. Mater. 2008, 20, 6310-6312. [CrossRef]

33. Park, Y.; Lee, J.H.; Jung, D.H.; Liu, S.H.; Lin, Y.H.; Chen, L.Y.; Wu, C.C.; Park, J. An aromatic imine group enhances the EL efficiency and carrier transport properties of highly efficient blue emitter for OLEDs. J. Mater. Chem. 2010, 20, 5930-5936. [CrossRef]

34. Gómez, R.; Veldman, D.; Langeveld, B.M.W.; Segura, J.L.; Janssen, R.A.J. A convergent synthesis of (diphenylvinyl)benzene (DPVB) star-shaped compounds with tunable redox, photo- and electroluminescent properties. J. Mater. Chem. 2007, 17, 4274-4288. [CrossRef]

35. Gómez, R.; Segura, J.L. Synthesis of a $\pi$-conjugated oligomer-fullerene dyad through a versatile [6,6]diphenylmethanofullerene carboxylic acid. Tetrahedron 2009, 65, 540-546. [CrossRef]

36. Iamazaki, E.T.; Atvars, T.D.Z. Sorption of a fluorescent whitening agent (tinopal CBS) onto modified cellulose fibers in the presence of surfactants and salt. Langmuir 2007, 23, 12886-12892. [CrossRef] [PubMed]

37. Yeh, A.T.; Shank, C.V.; McCusker, J.K. Ultrafast electron localization dynamics following photo-induced charge transfer. Science 2000, 289, 935-938. [CrossRef] [PubMed]

38. Gong, X.O.; Iyer, P.K.; Moses, D.; Bazan, G.C.; Heeger, A.J.; Xiao, S.S. Stabilized blue emission from polyfluorene-based light-emitting diodes: elimination of fluorenone defects. Adv. Funct. Mater. 2003, 13, 325-330. [CrossRef]

39. Zhang, Y.; Ye, X.; Petersen, J.L.; Li, M.; Shi, X. Synthesis and characterization of bis-N-2-aryl triazole as a fluorophore. J. Org. Chem. 2015, 80, 3664-3669. [CrossRef] [PubMed] 
40. Yan, W.; Wang, Q.; Lin, Q.; Li, M.; Petersen, J.L.; Shi, X. N-2-Aryl-1,2,3-triazoles: A novel class of UV/blue-light-emitting fluorophores with tunable optical properties. Chem. Eur. J. 2011, 17, 5011-5018. [CrossRef] [PubMed]

41. Wen, J.; Zhu, L.; Bi, Q.; Shen, Z.; Li, X.; Li, X.; Wang, Z.; Chen, Z. Highly N2-selective coupling of 1,2,3-triazoles with indole and pyrrole. Chem. Eur. J. 2014, 20, 974-978. [CrossRef] [PubMed]

42. Gu, C.-X.; Bi, Q.-W.; Gao, C.-K.; Wen, J.; Zhao, Z.-G.; Chen, Z. Post-synthetic modification of tryptophan containing peptides via NIS mediation. Org. Biomol. Chem. 2017, 15, 3396-3400. [CrossRef] [PubMed]

43. Liu, H.; Ding, H.; Zhu, L.; Wang, Y.; Chen, Z.; Tian, Z. A indole-trizole-rhodamine triad as ratiometric fluorescent probe for nanomolar-concentration level $\mathrm{Hg}^{2+}$ sensing with high selectivity. J. Fluor. 2015, 25, 1259-1266. [CrossRef] [PubMed]

44. Bayliss, N.S.; McRae, E.G. Solvent effects in organic spectra: dipole forces and the franck-condon principle. J. Phys. Chem. 1954, 58, 1002-1006. [CrossRef]

45. Seliskar, C.T.; Brand, L. Electronic spectra of 2-aminonaphthalene-6-sulfonate and related molecules. II. Effects of solvent medium on the absorption and fluorescence spectra. J. Am. Chem. Soc. 1971, 93, 5414-5420. [CrossRef]

46. Michl, J.; Thulstrup, E.W. Ultraviolet and infrared linear dichroism: polarized light as a probe of molecular and electronic structure. Acc. Chem. Res. 1987, 20, 192-199. [CrossRef]

47. Lobo, B.C.; Abelt, C.J. Does PRODAN possess a planar or twisted charge-transfer excited state? photophysical properties of two PRODAN derivatives. J. Phys. Chem. A 2003, 107, 10938-10943. [CrossRef]

48. Yoshihara, T.; Druzhinin, S.I.; Zachariasse, K.A. Fast intramolecular charge transfer with a planar rigidized electron donor/acceptor molecule. J. Am. Chem. Soc. 2004, 126, 8535-8539. [CrossRef] [PubMed]

49. Haas, E.; Katchalski-Katzir, E.; Steinberg, I.Z. Effect of the orientation of donor and acceptor on the probability of energy transfer involving electronic transitions of mixed polarization. Biochemistry 1978, 17, 5064-5070. [CrossRef] [PubMed]

50. Latt, S.A.; Cheung, H.T.; Blout, E.R. Energy transfer. A system with relatively fixed donor-acceptor separation. J. Am. Chem. Soc. 1965, 87, 995-1003. [CrossRef] [PubMed]

51. Giribabu, L.; Kumar, A.A.; Neeraja, V.; Maiya, B.G. Orientation dependence of energy transfer in an anthracene-porphyrin donor-acceptor system. Angew. Chem. Int. Ed. 2001, 40, 3621-3624. [CrossRef]

52. Nad, S.; Pal, H. Electron transfer from aromatic amines to excited coumarin dyes: fluorescence quenching and picosecond transient absorption studies. J. Phys. Chem. A 2000, 104, 673-680. [CrossRef]

53. Singh, M.K.; Pal, H.; Sapre, A.V. Interaction of the excited singlet state of neutral red with aromatic amines. Photochem. Photobiol. 2000, 71, 300-306. [CrossRef]

54. Wong, K.F.; Bagchi, B.; Rossky, P.J. Distance and orientation dependence of excitation transfer rates in conjugated systems: beyond the förster theory. J. Phys. Chem. A 2004, 108, 5752-5763. [CrossRef]

55. Yu, S.-Y.; Huang, H.-P.; Li, S.-H.; Jiao, Q.; Li, Y.-Z.; Wu, B.; Sei, Y.; Yamaguchi, K.; Pan, Y.-J.; Ma, H.-W. Solution self-assembly, spontaneous deprotonation, and crystal structures of bipyrazolate-bridged metallomacrocycles with dimetal centers. Inorg. Chem. 2005, 44, 9471-9488. [CrossRef] [PubMed]

56. Lehmann, M.; Marcos, M.; Serrano, J.L.; Sierra, T. Rigid chiral building blocks for copper(II)- and palladium(II)-containing liquid crystals. Chem. Mater. 2001, 13, 4374-4381. [CrossRef]

57. Espinet, P.; Lalinde, E.; Marcos, M.; Pérez, J.; Serrano, J.L. Liquid crystals based on ortho-palladated azines: Nonplanar mesogens. Organometallics 1990, 9, 555-560. [CrossRef]

58. Barberá, J.; Espinet, P.; Lalinde, E.; Marcos, M.; Serrano, J.L. New organometallic liquid crystals I. Orthopalladated imine derivatives. Liq. Cryst. 1987, 2, 833-842. [CrossRef]

Sample Availability: Samples of the compounds $3 \mathbf{a}-\mathbf{3 y}$ are available from the authors. 